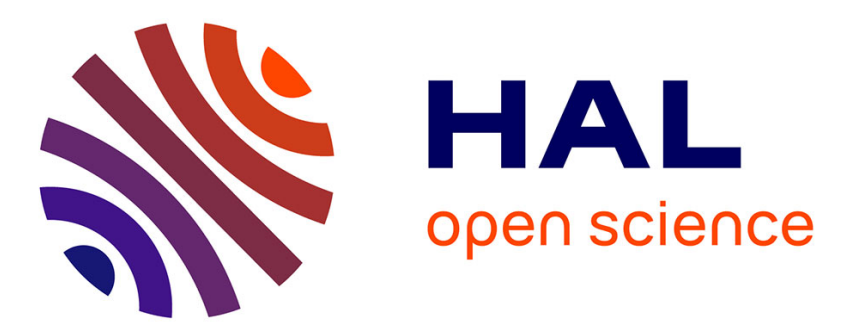

\title{
Impacts of geometric model simplifications on wave propagation-application to ground motion simulation in the lower Var valley basin (France)
}

Pierre Anquez, Nathalie Glinsky, Paul Cupillard, Guillaume Caumon

\section{- To cite this version:}

Pierre Anquez, Nathalie Glinsky, Paul Cupillard, Guillaume Caumon. Impacts of geometric model simplifications on wave propagation-application to ground motion simulation in the lower Var valley basin (France). Geophysical Journal International, 2022, 229 (1), pp.110-137. 10.1093/gji/ggab447 . hal-03578537

\section{HAL Id: hal-03578537 \\ https://hal.science/hal-03578537}

Submitted on 28 Feb 2022

HAL is a multi-disciplinary open access archive for the deposit and dissemination of scientific research documents, whether they are published or not. The documents may come from teaching and research institutions in France or abroad, or from public or private research centers.
L'archive ouverte pluridisciplinaire HAL, est destinée au dépôt et à la diffusion de documents scientifiques de niveau recherche, publiés ou non, émanant des établissements d'enseignement et de recherche français ou étrangers, des laboratoires publics ou privés. 


\title{
Impacts of geometric model simplifications on wave propagation - Application to ground motion simulation in the lower Var valley basin (France).
}

\author{
Pierre Anquez ${ }^{1,2}$ \\ Nathalie Glinsky ${ }^{3}$ \\ Paul Cupillard ${ }^{1}$ \\ Guillaume Caumon ${ }^{1}$
}

28 February 2022

\begin{abstract}
SUMMARY
The numerical simulation of seismic wave propagation in realistic heterogeneous media, as sedimentary basins, is a key element of seismic hazard estimation. Many numerical methods in two dimensions are based on unstructured triangular meshes and explicit time schemes. However, the presence of thin layers and tangential stratigraphic contacts in sedimentary basins entails poorly shaped mesh elements: some triangle heights are extremely small compared to the edge lengths, which requires small time steps in the simulations and thus leads to prohibitive computation times. We compare manual and automatic geological model simplification techniques to modify problematic areas of the domain, so as to improve the quality of the triangulated mesh. We modify the shape and the connectivity between rock units in the basin, with the objective to reduce the computation time without significantly changing the physical response of the geological medium. These simplification techniques are applied in an investigation of site effects in the lower Var valley, a densely urbanized area located near the city of Nice (SouthEast of France). Numerical simulations of plane wave propagation in a heterogeneous $2 \mathrm{D}$ profile are carried out with a discontinuous Galerkin finite element method. Five simplified meshes are generated and the impacts of the simplifications are analyzed in comparison to the reference model. We compare the time solutions and the transfer functions obtained on the surface of the basin. The results show that the simplification procedures, in particular automatic modifications of the model, yield a significant performance gain, with a ratio higher than 55, while having a negligible impact on the ground motion response.
\end{abstract}

keywords: Numerical modelling - Computational seismology - Earthquake hazards - Site effects - Composition and structure of the continental crust

This is a pre-copyedited, author-produced PDF of an article accepted for publication in Geophysical Journal International following peer review. The version of record (Geophysical Journal International, Volume 229, Issue 1, April 2022, Pages 110-137) is available online at: https:// doi. org/10.1093/gji/ggab447

\section{Introduction}

Seismic hazard assessment and in particular the estimation of ground motion at local scale are essential for mitigation policies. Indeed, seismic waves are modified, often amplified, by local geological and geotechnical conditions. These variations are known as site effects and are of two different types: lithological site effects in the case of resonance of seismic waves in shallow geological layers and topographical site effects in presence of irregular topographic features (?). Such a resonance amplifies some frequencies of the waves at the surface and extends the ground motion duration, which potentially increases the seismic impact on buildings and people. These phenomena have been widely studied for over sixty years (?). In this work, we focus on lithological site effects. Some results regarding the impacts of the topography on the ground motion can be found in ????) or the review proposed by ?), to only cite a few.

Lithological site effects in alluvial basins and sedimentary valleys have first been highlighted from recordings during strong and destructive events. For example, the Michoacán earthquake in Mexico (September 19, 1985) resulted in more than 5000 deaths and three billion US\$ in damage 
in Mexico City, although the epicenter was located at more than $350 \mathrm{~km}$ from the city. The site effects within the original lake basin on which the city is built explain this dramatic toll (??). Another example is the Loma Prieta earthquake that struck the San Francisco Bay area (October 17, 1989) and caused 63 deaths, nearly 3800 injuries and an estimated six billion US\$ in property damage. Measurements of the aftershocks in the alluvium-filled Santa Clara valley proved the role of surface waves generated at the basin edges in the peak velocities and displacements at some sites in the valley (?). Almost all recent destructive earthquakes have brought additional evidence of the impact of site effects. Many highly populated areas are located along river valleys or on alluvial basins, hence the importance of an accurate estimation of these effects.

Ground motion modeling has become a major component of seismic hazard assessment thanks to the constant improvement of physical models, numerical methods and computational resources. It is particularly useful in areas of low or moderate seismic hazard where ground motion data are limited. It requires a relevant geological model of the region of study, which is usually inferred from subsurface data such as geophysical images and borehole data. However, sedimentary basins often display sharp lithological and petrophysical discontinuities, owing to the presence of gaps at multiple scales during the depositional history (??). The geometry of the discontinuities to be accounted for in numerical modeling is, therefore, determined by the interfaces between rock units at some relevant scale based on geological and physical considerations (?). However, choosing which geological features can be represented by effective properties and which features should be handled explicitly is challenging (??). The homogenization theory (?) offers a way to rigorously address this problem for seismic wave propagation. It is based on series expansions of both the displacement and the stress fields. Unfortunately, in the current state of knowledge, the solution provided by this theory in the presence of an irregular free surface topography is limited to the order 0 , which can lead to a poor accuracy, especially for long-travelling surface waves (?).

Numerical studies of the seismic response of $2 \mathrm{D}$ and 3D sedimentary basins, considering different types of idealized geometries and some variable parameters, made it possible to explain qualitatively the seismic observations as well as the influence of some data such as the shape of the interface and the velocity contrast between the bedrock and the softer basin sediments (??????). From a quantitative point of view, however, simplified models do not necessarily allow to reproduce the observed amplification levels or the high spatial variability of the ground motion. Consequently, many numerical applications have studied the seismic response of more realistic $2 \mathrm{D}$ and $3 \mathrm{D}$ basin or valley models (????), some of which have benefited from a very detailed subsoil characterization (???????). As compared to simple models, complex, non-symmetrical basin shape and heterogeneity in the sedimentary part generate a greater variability of the motion at the surface and locally stronger amplifications, closer to what is observed on recorded data. In almost all cases, there are significant differences between the response of the $2 \mathrm{D}$ or $3 \mathrm{D}$ model at some points of the surface and the corresponding 1D case (i.e., the case of horizontally stratified layers or 1D soil column); this illustrates the limitations of simple "1D" approximations to produce reliable estimations in such configurations. Comparisons between simulations using 1D, 2D and 3D models at a same location lead to more nuanced conclusions (???). In particular, the differences between the response of $2 \mathrm{D}$ and $3 \mathrm{D}$ models are sometimes quite small, only quantitative, especially in elongated basins and valleys. Given the amount of data necessary to build an accurate 3D model as well as the computational cost of a 3D simulation, the study of the response of a $2 \mathrm{D}$ cross-section with a detailed subsoil description can then be a good compromise.

Many different numerical methods can be used for the simulation, among which we can cite the discrete wavenumber method (DWM, e.g. ?), the boundary element method (BEM, e.g. ????), the finite difference method (FDM, e.g. ????), the finite element method (FEM, e.g. ???), the pseudo-spectral method (PSM, e.g. ?), the spectral element method (SEM, e.g. ????????) and the discontinuous Galerkin method (DGM, e.g. ?????). All these techniques require a proper spatial discretization of the geological interfaces (i.e., a mesh) in order to accurately account for their effects on wave propagation. In the common case where uncertainties exist about the geometry of geological structures, this discretization should be computed on several possible geological models for the rigorous assessment of the nonlinear effects of geological uncertainties on the simulated wavefield (?). However, when the geometry of the interfaces is complex, obtaining even a single deterministic mesh can be a difficult task and the computation of wave propagation can be extremely costly. In particular, when the interfaces are close one to another, involving tangential contacts and thin geological layers, the mesh required in the finite-element type methods (FEM, SEM or DGM) can be challenging to generate. This mesh may contain a large number of elements and a few elongated elements, which results in prohibitive computational cost. Indeed, most of the methods are based on explicit time schemes, so the global time step of the simulation is the minimum local value in each mesh element. This value depends on the element size, the local compressional wave velocity $\left(V_{P}\right)$ and the interpolation degree of the method.

To deal with this difficulty, the DGM enables the socalled p-adaptivity, which consists in using a non-uniform polynomial degree distribution to relax constraints on the time-step in small elements (????). This method is all the more efficient when associated to non-conforming meshes, as explored for finite-element type methods (????), but the coupling between the coarse and fine non-conforming elements in this context can prove to be very complex, in particular for 3D meshes based on tetrahedra. Another solution for dealing with complex geometry consists in implementing hybrid methods to benefit from the advantages of one method in a given part of the space domain (e.g., the flexibility of the DGM where a complex geometry is involved) and of another method in the rest of the domain (e.g., the efficiency of the SEM where hexahedra can be easily handled). Hybrid methods include BEM-DWM (e.g. ?), FEMBEM (e.g. ?), FEM-FDM (e.g. ?), tetrahedral-hexahedral FEM (e.g. ?), FEM-SEM (?) and DGM-SEM (?). Most of these methods call for hex-dominant meshes, which can be challenging to generate (???).

In combination with any of the above solutions, a slight 
simplification of complex geometrical features is a possible strategy to make ground motion modeling tractable. For example, ?) apply a smoothing to cross-sections extracted from a 3D engineering-geological model, and ?) regularize the interfaces between materials of a $2 \mathrm{D}$ model by using spline functions to facilitate the generation of a triangular mesh. Such simplifications are probably widely used even when they are not mentioned. Whereas they probably have a limited impact for low frequencies, they may introduce inaccuracies in the solution for high frequencies. When performed manually, they may also involve decisions from the operators which are not necessarily documented. Moreover, manual and local mesh simplifications are cumbersome, so they cannot be efficiently applied to a large number of stochastic structural models reflecting geological uncertainties.

To overcome these issues, ?) proposed an automatic simplification technique based on exclusion areas and discussed the impact of this simplification on simulated wavefield in a quantitative way. In the present study, we propose to combine and compare a manual simplification strategy with the automatic method of ?) using the $2 \mathrm{D}$ model of a real site, and we investigate the effects of these simplifications in terms of computational cost and solution accuracy. The 2D model we choose for our study is a cross-section from the lower Var valley, of which a 3D detailed model was recently finalized by ?). The site effect estimation study we carry out is standard and applicable to other sites. Our 2D wave propagation simulations are performed using a DGM solver (?), but the simplification method which is proposed can be applied before other numerical simulation methods based on a triangular discretization.

The paper is organized as follows. In section 1, we present the application site and the available data, the initial $2 \mathrm{D}$ cross-section and the various components of the numerical method. Section 2 briefly explains and illustrates the simplification methods and details the five models generated from the initial one. The results are presented in section 3; first, the study of site effects using the initial model and then the comparison between the results obtained with simplified models. The computation times, as well as time and frequency solutions are compared.

\section{METHODS AND MODELS}

\subsection{Initial data}

The lower Var valley is the site chosen to apply and illustrate the method. This site, located west of the city of Nice (southeast France), is a highly urbanized fluvial sedimentary basin. It is an area in strong economic development which hosts the second largest French airport. Because it stands near the Ligurian margin and the Alps, this region presents a moderate but regular seismic activity. It is one of the rare areas in metropolitan France where the return period of earthquakes of magnitude greater than 4.5 is shorter than 10 years (e.g. ?). Several site specific studies have shown site effects in the city of Nice and the lower Var valley, its western boundary (?). For that reason, it is important to continue evaluating the seismic risk in this region.

A 3D subsurface model of the valley has been recently finalized by ?). This model is based on the compilation of sev-

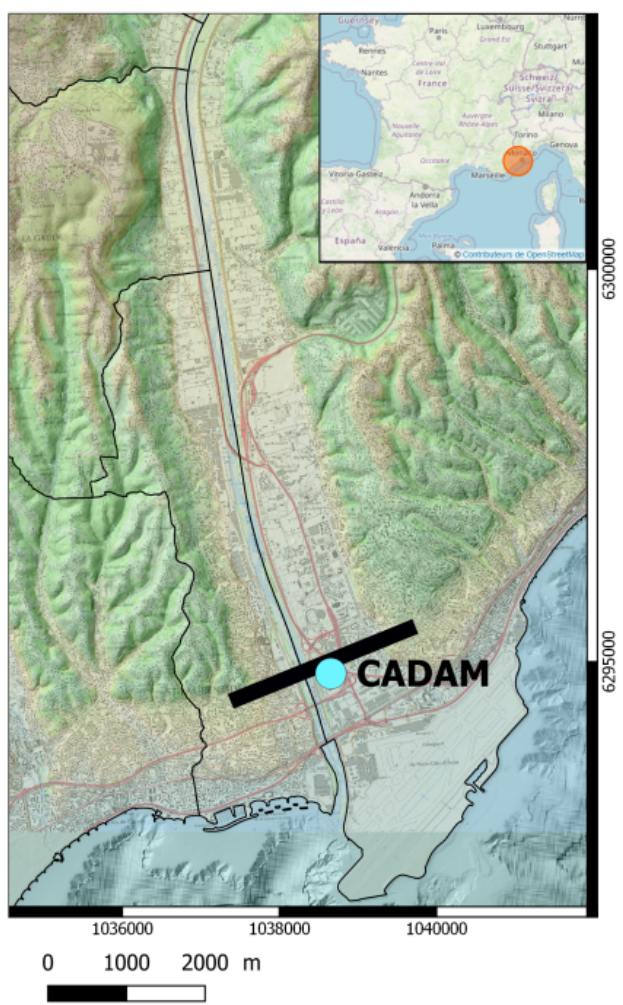

Figure 1. Extract of the lower Var valley map. The studied profile is located near the prefecture of Alpes-Maritimes county (CADAM).

eral years of geotechnical and geophysical studies and integrates data from several hundred borehole log-stratigraphies and ambient vibration measurements. These various data were combined in the GDM software (?) to build and constrain the 3D model of the lower Var valley, by interpolating the different interfaces between rock units. The result is a model describing the elevation of the geological interfaces and the geomechanical properties at depth on a regular grid with a $10 \mathrm{~m}$ resolution in both horizontal directions. The cross-section we investigate corresponds to a West-SouthWest/East-North-East profile at the level of the prefecture of Alpes-Maritimes county (CADAM), as depicted in Figure ??. The profile crosses the alluvial basin of the lower Var valley and connects from west to east the two cities of Saint-Laurent-du-Var and Nice.

The geometry of the cross-section is directly extracted from the 3D model. Its direction being not aligned with the model grid, the depth of the interfaces in our profile is known every 11.25 meters over 212 points. The total extension of the cross-section is about 2.37 kilometers.

The different materials which compose the geological section are presented in Figure ?? and are derived from the analysis of ?). An average value for the S-wave velocity, assumed as constant in each layer, is assigned to each rock unit according to the combination of different types of available data (geological knowledge, borehole data, $V_{S}$ profiles from inversion at some locations in the valley). Values for $V_{P}$ are deduced from the relationship $V_{P}=\sqrt{3} V_{S}$. For simplicity, a single value of density is defined in the basin: 


\begin{tabular}{|c|c|c|c|c|}
\hline Layer description & Code & Geology ( $f / 50000$ geological map) & $v_{p}\left(m s^{-1}\right)$ & $V s\left(m s^{-1}\right)$ \\
\hline $\begin{array}{l}\text { Filling, concrete. } \\
\text { Topsoil. }\end{array}$ & 1 & Anthropogenic deposits & 600 & 240 \\
\hline Sand, pebbles, gravels. & 2 & Recent alluvium & 1080 & 435 \\
\hline $\begin{array}{l}\text { Fine sand. Silty sand or } \\
\text { clay sand. }\end{array}$ & 3 & Deltaic alluvium & 875 & 350 \\
\hline $\begin{array}{l}\text { Silt, clay } \\
\text { (sometimes muddy). }\end{array}$ & 4 & Muddy deposits and pro-delta & 675 & 270 \\
\hline Sand, pebbles, gravels. & 5 & Recent alluvium, fluvial deposits & 1080 & 435 \\
\hline $\begin{array}{l}\text { Fine sand. Silty sand or } \\
\text { clay sand. }\end{array}$ & 6 & Deltaic alluvium & 875 & 350 \\
\hline $\begin{array}{l}\text { Silt, clay } \\
\text { (sometimes muddy). }\end{array}$ & 7 & Muddy deposits and pro-delta & 675 & 270 \\
\hline Coarse sand. & 8 & Ancient alluvium, fluvial deposits & 1125 & 450 \\
\hline Sand, pebbles, gravels. & 9 & Ancient alluvium, fluvial deposits & 1250 & 500 \\
\hline Bedrock. & $\mathrm{R}$ & Pliocene, Cretaceous, Jurassic, Trias & 2234 & 1290 \\
\hline
\end{tabular}

Figure 2. Nature and properties of the materials which compose the subsurface of the lower Var valley. Modified from ?).

$\rho_{\text {basin }}=1900 \mathrm{~kg} / \mathrm{m}^{3}$. The bedrock density is also considered as constant: $\rho_{\text {rock }}=2100 \mathrm{~kg} / \mathrm{m}^{3}$. In the basin, the shear wave velocities corresponding to the different types of alluvium vary between 240 and $500 \mathrm{~m} / \mathrm{s}$. The contrast with the bedrock $\left(V_{S}=1290 \mathrm{~m} / \mathrm{s}\right)$ is relatively high.

\subsection{Reference model (M1)}

An initial mesh that will serve as a reference is generated from the raw data of the $3 \mathrm{D}$ model. The geological interfaces between materials, as well as the top and the base of the basin, are first constructed by linking the data points of the regular grid. The obtained lines are then smoothed using cubic splines. The top topography corresponds to the upper boundary of the numerical domain. The bottom of the cross-section has a constant depth of 123 meters. In the lateral direction, the homogeneous bedrock is extended artificially (Figure ??). The extension aims to gradually bring the two sides of the model to the same constant thickness (elevation fixed at 65 meters) so that periodic conditions on the lateral boundaries can be applied. In summary, the final model dimensions are 3700 meters by 188 meters, the basin having a lateral extension of about 1300 meters and a maximum depth of about 50 meters (Figure ??).

A first triangulated unstructured mesh of the geological section was generated using the mmg2D softwarf*?(??). The target mesh element sizes were set to 1 meter in the basin and 4 meters in the bedrock far from the basin. In the bedrock, the size of the mesh elements gradually increases from 1 meter close to the basin interface to 4 meters. Within the basin, the interfaces between the materials are honored by the mesh. This mesh contains elongated triangles with small angles located at tangential contacts and in thin layers within the basin. Due to their small height, one can expect that these triangles have an influence on the time step and therefore the computational time of the simulations.

\subsection{Wave propagation simulations}

The study of the seismic response of the 2D profile is carried out by considering the $P-S V$ wave propagation of a vertical shear plane wave, from the depth to the surface through the heterogeneous basin. Simulations are performed with the discontinuous Galerkin finite element method (DGM) described in ?), for which we give the key elements below. The medium is supposed to be viscoelastic. The elastodynamic equations written in velocity-stress formulation are coupled to the GMB model (Generalized Maxwell Bodies) introduced by ?) using three mechanisms. The medium properties are supposed to be constant in each element of the triangular finite element mesh which requires the interfaces between the different geological materials to be represented as mesh boundaries. The spatial interpolation is local to each element and based on first order Lagrange polynomials. A centered flux is applied at the interfaces between adjacent mesh elements. Time integration uses a leap-frog scheme.

In the absence of information regarding attenuation in the different materials, the values of the quality factors $Q_{P}$ and $Q_{S}$ are assumed to be frequency independent and are estimated as one tenth of the $P$ - and $S$-wave velocities respectively, as frequently done in site effect studies where no other constraint on intrinsic attenuation is available (?). Refined relationships for the quality factors can be applied from ?). A periodicity condition is prescribed on the lateral boundaries for a more accurate propagation of the plane wave in the bedrock. A free surface condition is set at the topography. The shear incident plane wave is introduced at the base of the model via an upwind boundary flux (balance condition between incident and outgoing waves), following the technique described in ?). In this way, unlike when generating a plane wave by a line of point sources, the incident wave does not depend on the mesh in the bedrock, which is a necessary condition for an accurate comparison between the different model solutions. In this study, one propagates a wave whose horizontal velocity pattern is presented in Figure ?? (left). According to the normalized Fourier spectrum of Figure ?? (right), the energy is maximum up to $15 \mathrm{~Hz}$ and then decreases up to $25 \mathrm{~Hz}$. The minimum wavelength $\lambda_{\min }=V_{s} / f_{\max }$, for $f_{\max }=25 \mathrm{~Hz}$, is equal to 10 meters in the basin and 51 meters in the bedrock; the element size (i.e., maximum 1 meter in the basin and 4 meters in the bedrock) is therefore sufficient. The duration of the simulation is $T=4$ seconds, the time after which the wave energy is dissipated.

The medium response is recorded at 148 receivers placed on the model surface every 25 meters. We particularly focus on six of them, referred as R1 to R6 in Figure ??. R1 and R6 are located outside the basin, and R2, R3, R4 and $\mathrm{R} 5$ are on the basin topography at variable elevations. Receiver R3 corresponds to the location of the CADAM (the administrative center of the Alpes-Maritimes county). We are interested in the time evolution of the horizontal $V_{x}$ and vertical $V_{z}$ components of the ground motion velocity as well as the transfer functions. The transfer function or standard spectral ratio (SSR) at a site is the spectral ratio of the horizontal velocity, i.e., the ratio in the frequency domain of the computed velocity at the site by the equivalent velocity at a flat surface of a rocky homogeneous medium. The standard spectral ratio technique is widely applied to the analysis

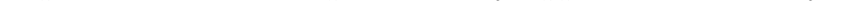




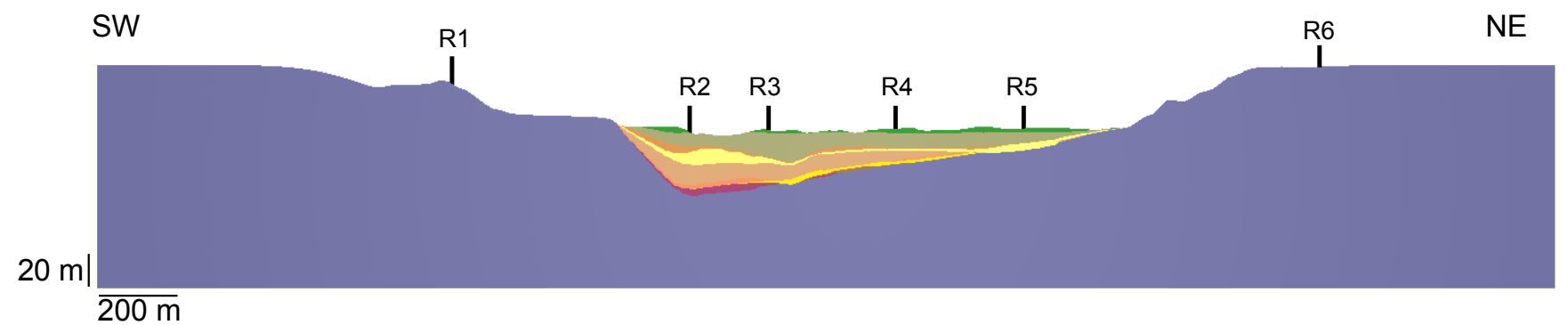

Figure 3. 2D geological section of the basin of the lower Var valley. The position of the 6 receivers is indicated on the profile: R1 and R6 are located outside the basin; R2, R3, R4 and R5 at the basin surface. R3 corresponds to the position of the CADAM on Figure ??. Vertical exaggeration $=3$.

of seismological recordings to better quantify the amplifications of a site relative to a reference (???). These ratios give indications on the level of amplification and the associated frequencies by normalizing with respect to the source. For these reasons, numerical applications on real sites are interested in transfer functions.

Because the solver is explicit in time, the global time step of the simulation must be chosen so as to guarantee computational stability. It writes

$$
\Delta t=\min _{i} \Delta t_{i}=\min _{i}\left[\frac{1}{2 p+1} \frac{H_{m i n} / i}{V_{P / i}}\right],
$$

where the index $i$ refers to the mesh element $i, p$ is the spatial interpolation degree (here $p=1$ ), $H_{m i n / i}$ and $V_{P / i}$ are respectively the minimum height and the compressional velocity $V_{P}$ of element $i$. The time discretization therefore depends on the smallest ratio between the minimum height of triangles and the corresponding $\mathrm{P}$-wave velocity. Because of poor quality triangles, the minimum height can be much smaller than the target element size imposed during the mesh construction, yielding a very small time step. To increase the time step and consequently decrease the computational cost, we propose to apply different techniques for simplifying the geometry of the cross-section. We compare five simplified models whose meshes have improved quality elements (Section ??).

\section{MODEL SIMPLIFICATION}

\subsection{Overview of the simplification method}

In this section, we shortly present the method for automatically simplifying $2 \mathrm{D}$ models we use in this study. Following the terminology of ?), these models consist of Surfaces (rock units) bounded by Lines (interfaces such as horizons and faults), which terminate or branch at Corners. The objective of the simplification is to decrease simulation time by reducing the number of mesh elements and by increasing their quality. In our case, computation time is much related to the smallest triangle height. Complex areas are thus those where Surfaces are thin, Lines are short or branch with small angles. The simplification is achieved by removing these small geometric features given some prescribed tolerances.

The automatic simplification method used in this study (?) takes as input two geometrical parameters: the minimal accepted distance between interfaces and the minimal accepted angle between interfaces. These two parameters are used as criteria to define invalid features of the model, in other words the areas where the two criteria are not verified. These criteria may be different from one model interface to another, but in this study, they are assumed to be the same everywhere in the model.

To detect invalid areas and features, all the 2D model interfaces, typically the lines between media and the contact points between these lines, are thickened similarly to the morphological dilation as defined in mathematical morphology (????). These dilations, called exclusion zones (?), are associated to each model entity and are defined using input geometrical criteria. If these exclusion zones overlap, the area where the overlap occurs does not fulfill input geometrical criteria and thus is considered invalid.

The geometrical analysis of model validity is based on exclusion zones. Exclusion zones are compared by pairs: each pair of intersecting exclusion zones are translated into an edge in an invalidity graph. Additional information are set on the invalid edge associating two model entities, mainly to localize with precision invalid areas.

Finally, the detected invalid features are repaired by choosing between two strategies. The first one uses expansion and only results in geometrical modification of model interfaces (Fig. ??-left). As a consequence, the topology of the model (i.e., the set of adjacency and incidence relations between Surfaces, Lines and Corners) is not modified. The second strategy is based on contraction that modifies both the geometry and the topology of the model by collapsing some model entities into lower dimensional entities (e.g., Lines into Corners, or Surface subsets into Lines, Fig. ??right). This strategy first operates topological modifications by applying a graph editing step to remove invalidity edges, and then reconstructs the repaired geometry of the geological model. After the simplification of the model interfaces, the model is remeshed. In this work, we use mmg2D for remeshing the simplified geometry. A more detailed description of this technique can be found in ?).

\subsection{Simplified models}

We generated five simplified versions, noted M2 to M6, of the reference cross-section (M1). The objective is to increase the minimal height of the triangles. The ambition is to reduce the computation time of the simulations. Special attention is therefore paid to thin layers and tangential contacts in the cross-section (Figure ??). All the meshes corresponding 

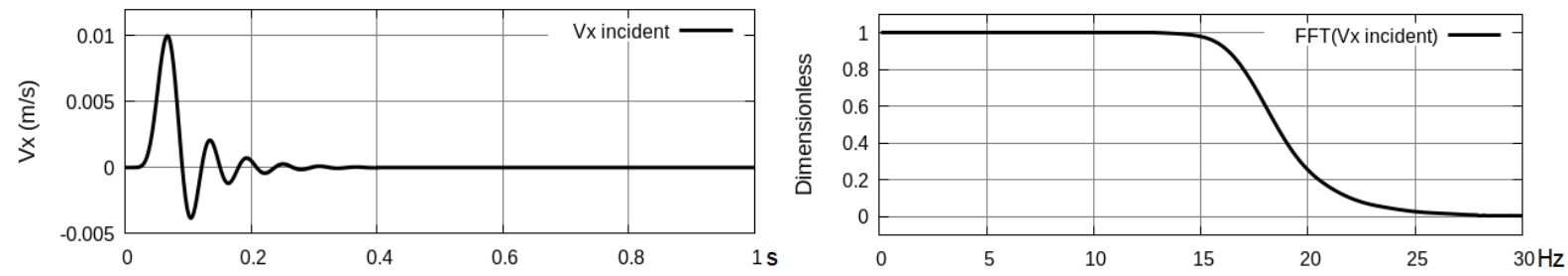

Figure 4. Time evolution of the horizontal velocity (left) and normalized Fourier spectrum (right) of the incident plane wave.

to the various models are provided in .msh format as supplementary material, freely available on GitHub (see ??).

To more easily link a model to the type of simplification used, we introduce the following notations: $\operatorname{Sm}(\mathrm{M})$ and $\mathrm{Sa}(\mathrm{M})$ denote respectively the manual or automatic simplification of the model M. Moreover, we distinguish by the additional notations $*$, t and ${ }^{*}, \mathrm{~g}$ the simplifications which modify the topology from those which modify only the model geometry.

Two groups of simplified models are defined: first the models M2, M3 and M4, which are modified with a manual simplification, and second the models M5 and M6 which are exclusively based on automatic simplifications as presented in Sec. ??. Table ?? gives information on which method is employed with parameter values for each simplified model. For all models, meshing target sizes are the same: 1 meter in the basin and 4 meters in the bedrock, which yields similar numbers of mesh elements for all the models. Table ?? summarizes for each model the number of model entities (Corners, Lines and Surfaces) as well as the number of mesh elements.

Model M2, named Sm,t(M1), comes from a manual simplification of the reference model. The simplifications involve several local editing steps based on visual criteria of the model complexity. The thinnest layers are removed locally or entirely, decreasing the number of model surfaces (Table ??). Moreover, tangential contacts between interfaces are enlarged to reduce small angles. Then, smoothing operations are performed on interface Lines. The smallest triangle height $H_{\min }$ is enlarged by a factor 8.2 , from $H_{\min }=2.77 \times 10^{-3} \mathrm{~m}$ for M1 to $2.27 \times 10^{-2} \mathrm{~m}$.

Model M3, or Sa,g(M2), is simplified from model M2 by applying the automatic method (?) using the expansion strategy (Sec. ??). We arbitrarily chose relatively large parameters for the exclusion zones, as model M2 was already relatively far from model M1. The smallest triangle height $H_{\min }$ is enlarged by a factor of 63.5 with respect to the reference model (from $H_{\text {min }}=2.77 \times 10^{-3} \mathrm{~m}$ to $1.76 \times 10^{-1} \mathrm{~m}$ ) corresponding to an enlargement factor of about 7.7 compared to model M2. Model M3 is the simplified model in which the smallest triangle height has been enlarged the most.

Model M4 (Sm,t(M2)) is also obtained from model M2, by removing the interface between layers 8 and 9 (Figure ??), which have similar rock properties and locally have a small thickness. These layers are the deepest of the basin, located between receivers $\mathrm{R} 2$ and $\mathrm{R} 4$. The velocity properties of material 8 are chosen for the merged entity. The smallest triangle height $H_{\min }$ is enlarged by a factor of $12.1 \mathrm{com}-$ pared to the reference model (from $H_{\text {min }}=2.77 \times 10^{-3} \mathrm{~m}$ to $3.35 \times 10^{-2} \mathrm{~m}$ ), a factor of 1.5 with respect to model M2.
Model M5, also named Sa,t(M1), is generated directly from model M1 by applying the automatic method presented in ?) using the contraction strategy. For this model, we chose relatively small simplification parameters (exclusion zones equal to $18 \%$ of the average edge size) to preserve geometric accuracy. The automatic processing had to be launched two times to remove all the small components remaining as artifacts in the model (for example, areas of length smaller than 0.5 meters). Indeed, two small components (thin layers) were still remaining after the first simplification run, particularly where the model M1 shows several and close small features. The second run, with same parameters, eliminated these artifacts without altering already simplified features in the model. The smallest triangle height $H_{\min }$ is enlarged by a factor of 31.4 compared to the reference model (passing from $H_{\text {min }}=2.77 \times 10^{-3} \mathrm{~m}$ for M1 to $8.71 \times 10^{-2} \mathrm{~m}$ ).

To improve the elements with small height in M5, the model M6 (also Sa,g(M5)) is generated from the model M5 by applying a second automatic simplification step using the expansion strategy (as for the generation of M3 from M2). In this case, we use the same geometric tolerance but a larger angle, as this makes it possible to thicken some layers in areas where three or more horizons were initially too close. The smallest triangle height $H_{\min }$ is enlarged by a factor of 53.4 compared to the reference model (from $H_{\text {min }}=2.77 \times 10^{-3} \mathrm{~m}$ to $1.48 \times 10^{-1} \mathrm{~m}$ ). As the parameter values for models M5 and model M6 are smaller than those used to generate M3 from M2 (Table ??), both models are geometrically closer to the reference model. The model M6, resulting from two simplifications, cannot be further than $0.36 \mathrm{~m}$ from the reference model M1.

\subsubsection{Geometric model comparison}

A random sampling of the models is performed to locate and estimate the amount of modifications introduced by both manual and automatic simplifications. For each pair of models, ten million points are randomly drawn in the basin area. The models are not sampled through a regular grid because the spacing required to capture the smallest changes would be of the order of $1 \mathrm{~mm}$, which would be prohibitive. The percentage of points that sample two different materials in two distinct models gives an approximation of the level of spatial differences between two models (Table ??). This spatial difference indicator is not applied to compare model M4 to other models, because M4 has been generated by removing an interface between two layers, which is not the same kind of modifications as all the other ones. The number of points was chosen to be large enough to ensure the statistical robustness of the percentage computation.

The differences of the modified models to the initial 
Strategy 1 : Expansion

(a)

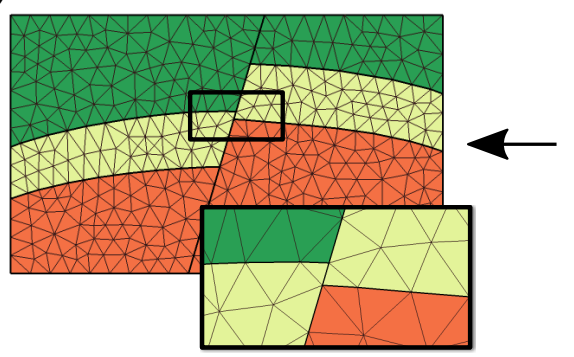

(b)

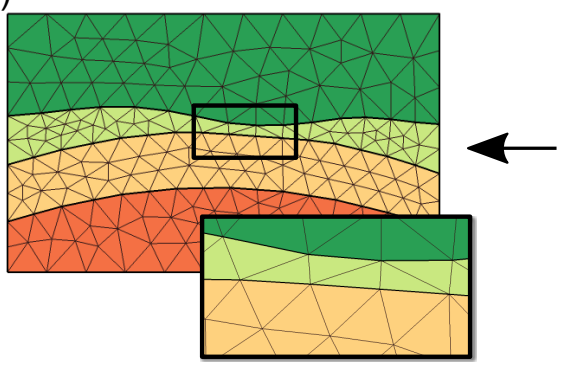

Strategy 2 : Contraction
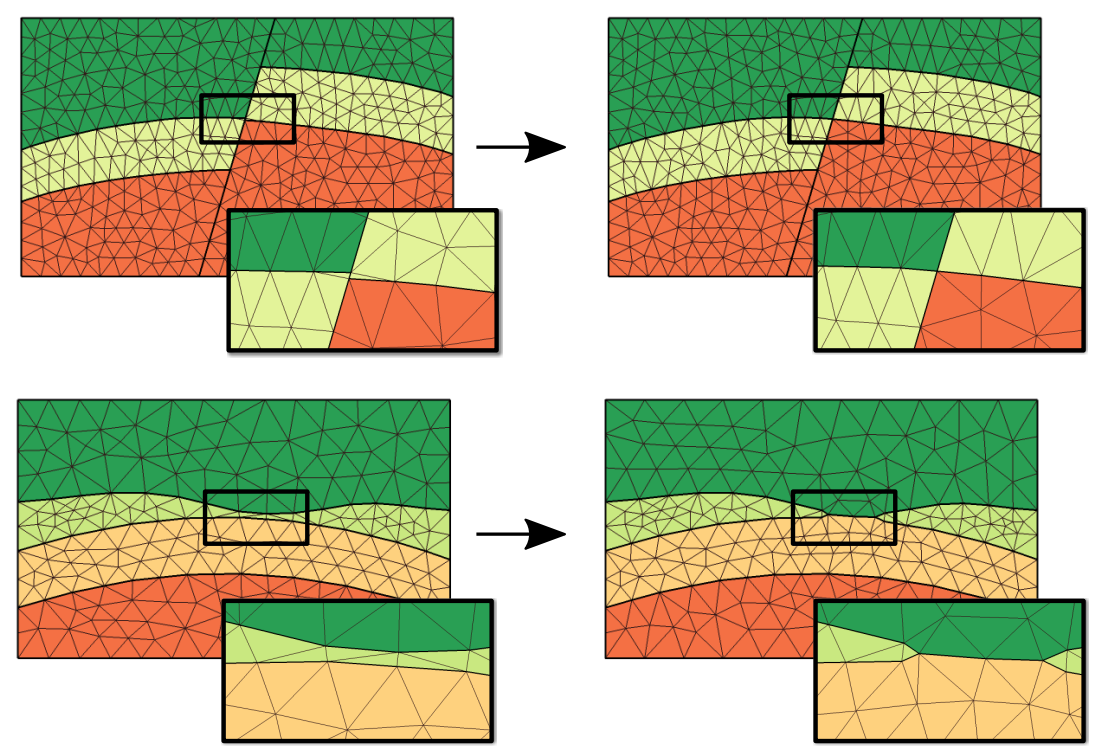

Figure 5. Illustration of the two automatic simplification strategies on a small geometric feature due to a fault (a) or a thin layer (b). From the initial model (center column), the expansion strategy (left) preserves the model connectivity whereas the contraction strategy (right) allows for topological changes during model simplification. Modified from ?).

Table 1. Summary of applied methods and parameters used to generate simplified models. For model notation, $\operatorname{Sm}(\mathrm{M})$ and $\mathrm{Sa}(\mathrm{M})$ refer respectively to manual and automatic simplifications applied to the model M. Addition indices $*, t$ and $*, g$ distinguish modifications in the topology from modifications of the geometry only. When the automatic method (?) is employed, simplification parameters are given in the right part.

\begin{tabular}{|c|c|c|c|c|c|c|}
\hline Simplified model & Model notation & Automatic method & Other method & Applied strategy & Minimal distance & Minimal angle \\
\hline M2 & $\mathrm{Sm}, \mathrm{t}(\mathrm{M} 1)$ & • & $\mathrm{X}$ (manual) & • & $\bullet$ & $\bullet$ \\
\hline M3 & $\mathrm{Sa}, \mathrm{g}(\mathrm{M} 2)$ & $\mathrm{X}$ & $\bullet$ & Expansion & $0.6 \mathrm{~m}$ & $15^{\circ}$ \\
\hline M4 & $\mathrm{Sm}, \mathrm{t}(\mathrm{M} 2)$ & $\bullet$ & $\mathrm{X}$ (unit merging) & $\bullet$ & $\bullet$ & $\bullet$ \\
\hline M5 & $\mathrm{Sa}, \mathrm{t}(\mathrm{M} 1)$ & $\mathrm{X}$ & $\bullet$ & Contraction & $0.18 \mathrm{~m}$ & $5^{o}$ \\
\hline M6 & $\mathrm{Sa}, \mathrm{g}(\mathrm{M} 5)$ & $\mathrm{X}$ & $\bullet$ & Expansion & $0.18 \mathrm{~m}$ & $10^{\circ}$ \\
\hline
\end{tabular}

Table 2. Model and mesh characteristics for models M1 to M6; the correspondence between the model numbers and the simplifications methods can be found in Table ??. The number of mesh elements (triangles) is comparable for all models. For each mesh, the minimum triangle height $H_{\min }$ is given, pointing out the quality of the worst mesh element. Enlargement ratio is the ratio between the value of $H_{\text {min }}$ of the current model and that of the reference model (M1).

\begin{tabular}{lcccccc}
\hline Model & M1 & M2 & M3 & M4 & M5 & M6 \\
\hline Nb. of Corners & 39 & 30 & 30 & 28 & 38 & 38 \\
Nb. of Lines & 61 & 44 & 44 & 41 & 56 & 56 \\
Nb. of Surfaces & 23 & 15 & 15 & 14 & 19 & 19 \\
\hline Nb. of vertices & 112007 & 110434 & 112123 & 110112 & 111294 & 111294 \\
Nb. of triangles & 221089 & 217943 & 221326 & 217304 & 219675 & 219675 \\
\hline$H_{\text {min }}(\mathrm{m})$ & $2.77 \times 10^{-3}$ & $2.27 \times 10^{-2}$ & $1.76 \times 10^{-1}$ & $3.35 \times 10^{-2}$ & $8.71 \times 10^{-2}$ & $1.48 \times 10^{-1}$ \\
Enlargement ratio & 1 & 8.2 & 63.5 & 12.1 & 31.4 & 53.4 \\
\hline
\end{tabular}



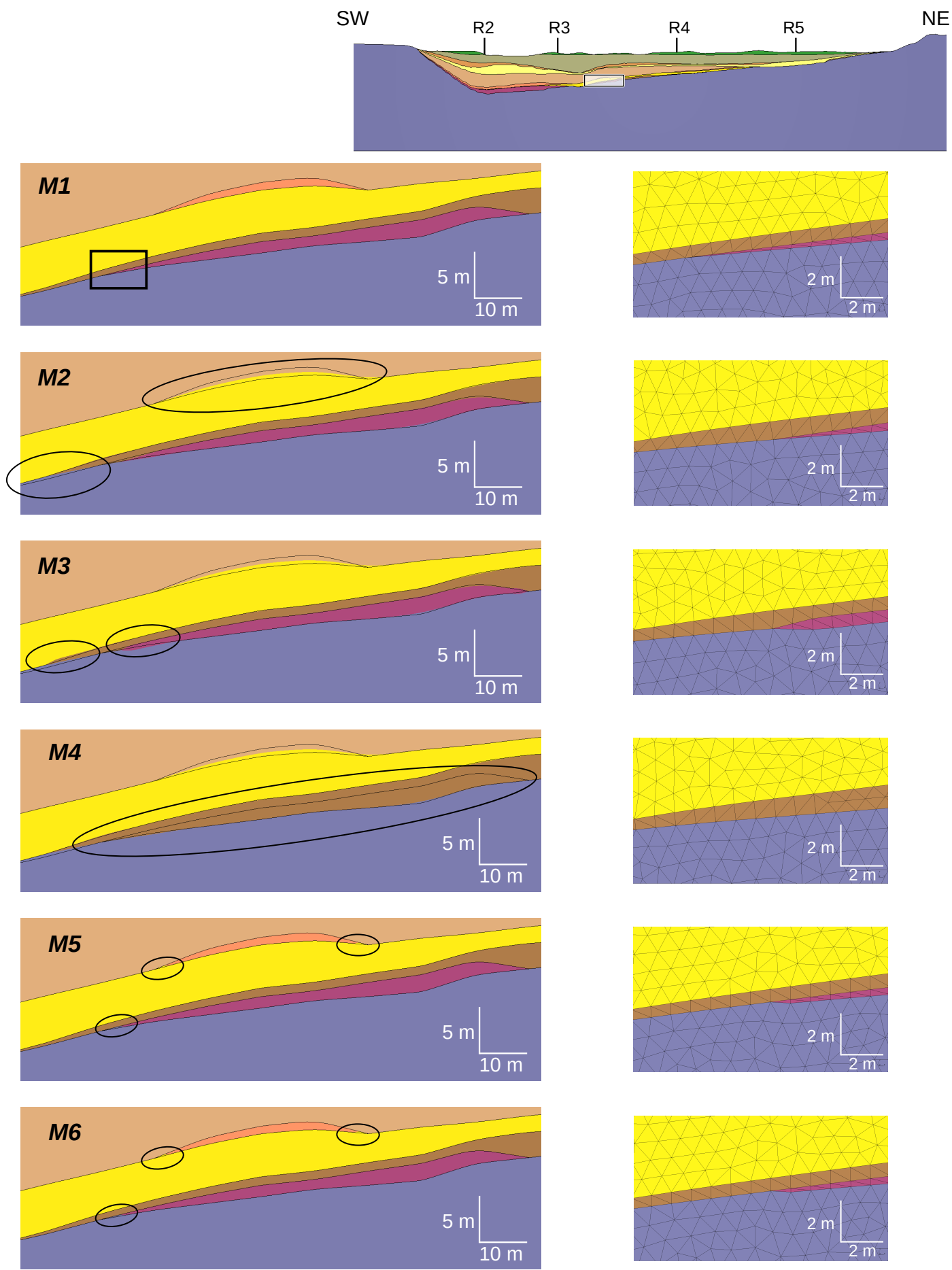

Figure 6. Detailed view of the reference model (M1) and the five simplified models (M2 to M6; the correspondence between the model numbers and the simplifications methods can be found in Table ??). For figures in the left side, black lines correspond to the interfaces in the reference model whereas colors correspond to materials in the simplified model (vertical exaggeration $=2$ ). The black square on M1 locates the detailed views on the right column; black ellipsoids helps to see changes. For figures in the right side, mesh elements are shown on a detailed view without vertical exaggeration. See Figure ?? for legend. 


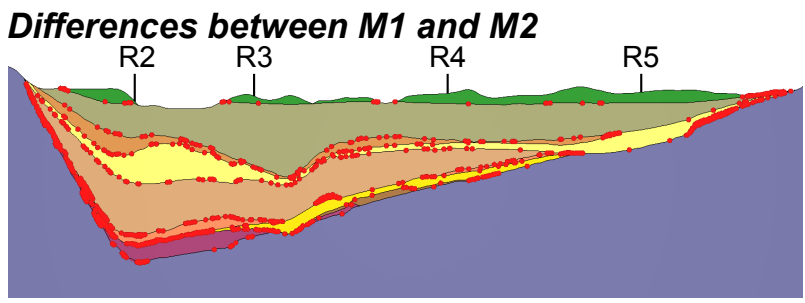

\section{Differences between M1 and M3}

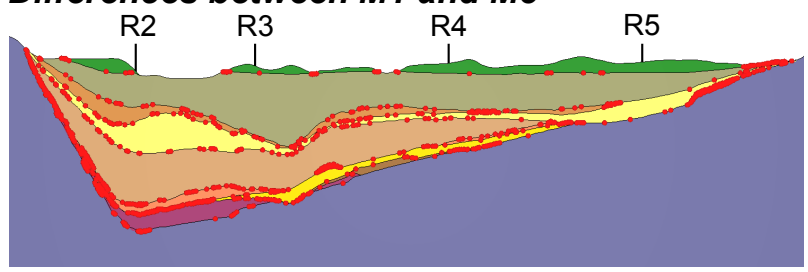

Differences between M1 and M5
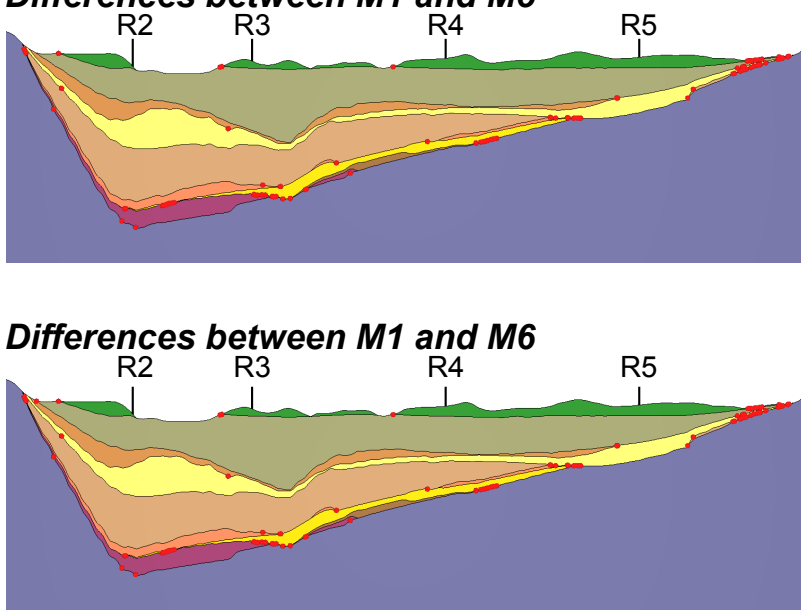

\section{Differences between M2 and M3}

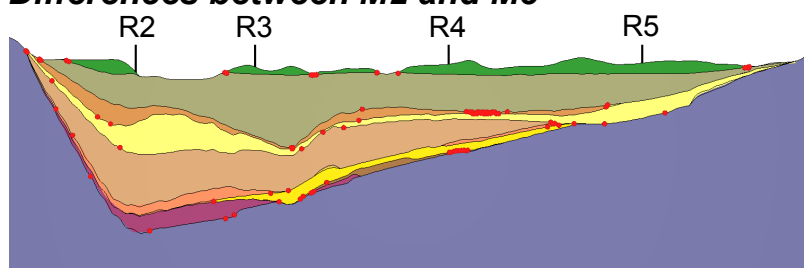

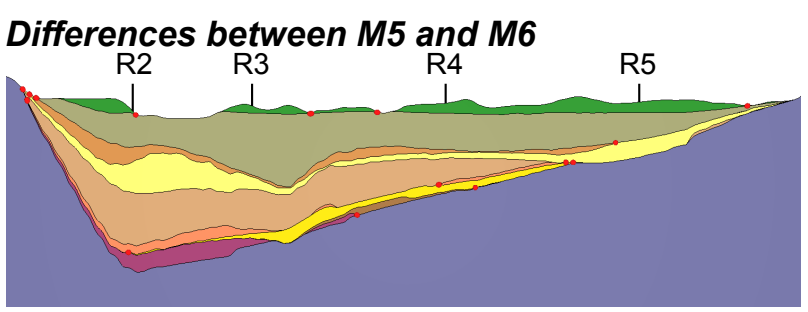

Figure 7. Localization of modified areas between pairs of models; the correspondence between the model numbers and the simplifications methods can be found in Table ??. Left : Differences between the reference model and the simplified models. Right : Differences between a model and the one from which it is derived. The red dots represent the points, among those randomly drawn in the basin, located in different materials. 100,000 points are randomly drawn in the basin for illustrating this figure, except for the comparison between M5 and M6 in which 10,000,000 points are used (100 times more). Vertical exaggeration $=5$.

Table 3. Estimation of differences between the models M1, M2, M3, M5 and M6. Values represent the percentage of incorrect rock units among ten million randomly sampled points.

\begin{tabular}{lcccc}
\hline & M1 & M2 & M3 & M5 \\
M1 & - & 1.32 & 1.40 & $1.12 \times 10^{-1}$ \\
M2 (Sm,t(M1)) & - & - & $1.37 \times 10^{-1}$ & 1.21 \\
M3 (Sa,g(M2)) & - & - & - & 1.33 \\
M5 (Sa,t(M1)) & - & - & - & - \\
M6 (Sa,g(M5)) & - & - & - & -
\end{tabular}

model M1 are more abundant for the model M2, and consequently for model M3, than for models M5 and M6 (Figure ??, Table ??). Differences between M1 and M2 exist on almost all the interfaces in the basin. This is due to the manual modifications applied to some interfaces of the reference model and to the smoothing operations. The highest density of changes (emphasized by a high density of red dots) is near the edges of the basin and at depth where the layers are the finest in the reference model. The differences between M1 and M5 are mainly localized near the tangential contacts in the basin and the thinnest layers, which illustrates the local 1.14 and ${ }_{0}$ minimal nature of the modifications performed by the automatic simplification method. Most of the differences lie in the North-East end of the basin and at the bottom of the b3asin just below receiver $\mathrm{R} 3$.

$2.20 \times 10^{-3}$ The comparisons between M2 - M3 and M1 - M5 reveal some isolated points located at interfaces in the basin far from areas of geometrical complexity (Figure ??). These points are caused by the remeshing step. During this process, the interfaces are slightly modified, involving changes about $10^{-3}$ meters large, which is very small compared to the scale of simplifications. However, these changes fall within observable variations between two models. They could, therefore, have an impact on numerical simulations, although they are very small in comparison to geometrical simplifications. 
Since the generation of M6 from M5 does not involve a remeshing of the model Lines and Surfaces, the differences are comparatively very small (Table ??) and they are located at the enlarged tangential contacts only.

\section{RESULTS}

In this section, we first present a study of the wave amplification in the lower Var valley basin obtained with the reference model M1. Then, we analyze the effects of the model simplifications on the numerical simulations. We first consider the base case with attenuation, and then analyze results for a simplified elastic case with no attenuation. We focus on two aspects: the reduction in computation time induced by the simplifications and the modifications of the numerical results due to these simplifications.

\subsection{Site effects study using the reference model M1}

In Figure ??, we present the numerical results of the vertical propagation of a shear plane wave, for all the surface receivers. For better visibility, the seismograms of both velocity components are plotted up to 2 . s. The position of receivers $\mathrm{R} 1$ to $\mathrm{R} 6$, located at respective abscissas of -1002 , $-400,-200,125,400$ and $1201 \mathrm{~m}$, is represented by red squares in the figures. On the seismograms (Figure ??-a-b), trapped waves inside the basin are observed, due to the material property contrast between the bedrock and the basin. The multiple reflections and refractions in the different layers of the basin generate complex patterns and non-zero vertical ground velocity values in the $\mathrm{x}$-interval $[-600,700]$ corresponding to the basin location. In Figure ??-c, we plot the transfer functions of the horizontal velocity at the surface, that is the spectral (Fourier) velocity ratio as a function of the frequency at each receiver. These ratios are calculated with respect to the reference surface response of a flat homogeneous bedrock. The spectra come directly from time solutions up to $4 \mathrm{~s}$; no filtering was applied in this study. Strong amplifications, with values up to 5 in the basin, are observed at a fundamental resonance frequency between 2 $\mathrm{Hz}$ and $5 \mathrm{~Hz}$ depending on the depth of the interface of highest velocity contrast. Large amplifications are also noticed at higher frequencies (between 10 and $15 \mathrm{~Hz}$ ) at receivers located in the eastern part of the basin.

The solutions obtained at the six receivers $\mathrm{R} 1$ to $\mathrm{R} 6$ are described in Figure ??. For R1 and R6, which are located outside the basin, the time evolution of the two ground velocity components (Figure ??-left-center) is roughly twice the amplitude of the incident signal (as expected at the free surface), and the transfer functions (Figure ??-right) are constant and close to 1 . In contrast, the basin effects are visible from R2 to R5. A clear amplification of the horizontal velocity is observed, especially in R4 and R5 (with a Peak Ground Velocity (PGV) equal to $3.0 \times 10^{-2} \mathrm{~m} . \mathrm{s}^{-1}$ ) and non-zero values of the vertical velocity are observed. We also notice an increase of the duration of the seismograms: they are about three times longer than the incident signal. Moreover, the transfer functions show an amplification up to 4 at about $2 \mathrm{~Hz}$ for $\mathrm{R} 2$ and $\mathrm{R} 3$, which correspond to the thickest part of the basin, and at higher frequencies for R4
(3Hz) and R5 $(4 \mathrm{~Hz})$ as the sediment thickness reduces. Several significant amplification peaks at higher frequencies (between 10 and $22 \mathrm{~Hz}$ ) are also observed at R3, R4 and $\mathrm{R} 5$. The fundamental frequency computed in R3 is in accordance with the results of SSR (Standard Spectral Ratios) and HVSR (Horizontal over Vertical Spectral Ratios) obtained by ?) at the CADAM, which both provide a first frequency peak at $1.8 \mathrm{~Hz}$. These are typical characteristics of site effects in sedimentary basins.

\subsection{Comparison of computation times}

The computation time necessary for the simulation of the plane wave propagation in the reference model M1 until 4 seconds is greater than 12 days (300 hours, Table ??) with a classic desktop computer. Indeed, the time step $\Delta t$ is chosen equal to $10^{-7}$ seconds to verify the stability criterion given in Eq.(??). In our simulations, the time step is controlled by the smallest ratio in the entire mesh between the minimum height of a triangle and the local value of the $\mathrm{P}$-wave velocity. The solver used in this study is not parallelized because the computation times are usually reasonable for $2 \mathrm{D}$ applications. The model M1, generated from raw data, is only used here to serve as a reference when comparing with the simplified models.

When the simplified models are used, the computation times decrease from 28 hours for model M2 to slightly more than 4 hours for model M3 (Table ??). Automatic crosssection simplifications take only a few seconds and remeshing adds no more than a few minutes on a standard desktop computer, so geometric processing time is negligible. Overall, the gain in computation time is considerable for all the simplified models, the CPU time ratio varying between 10 and 73 depending on the model. From the comparison of computation times, we can draw the following conclusions:

- Starting from the reference model, the application of automatic simplifications (generation of M5) results in smaller computation times than the simplifications by hand (generation of M2); the ratio between the calculation times of M5 and M2 is greater than 3.

- The application of additional automatic simplifications based on the expansion of thin layers and tangential contacts (models M3 and M6) leads to an additional gain in computation time; both models M3 and M6 correspond to the fastest computations.

- The simple merger of two layers in a specific area (generation of M4 from M2) reduces the computation time by half. However, this simplification method is generally not optimal and must be applied with care, as it removes an entire interface so it affects areas that have no impact on the calculation time and that could be kept intact (e.g., the thickest part of layer 9, far from the edges; see Figure 5). Conversely, it does not modify the rest of the model, leaving unchanged areas that can have a strong influence on computational performance. 


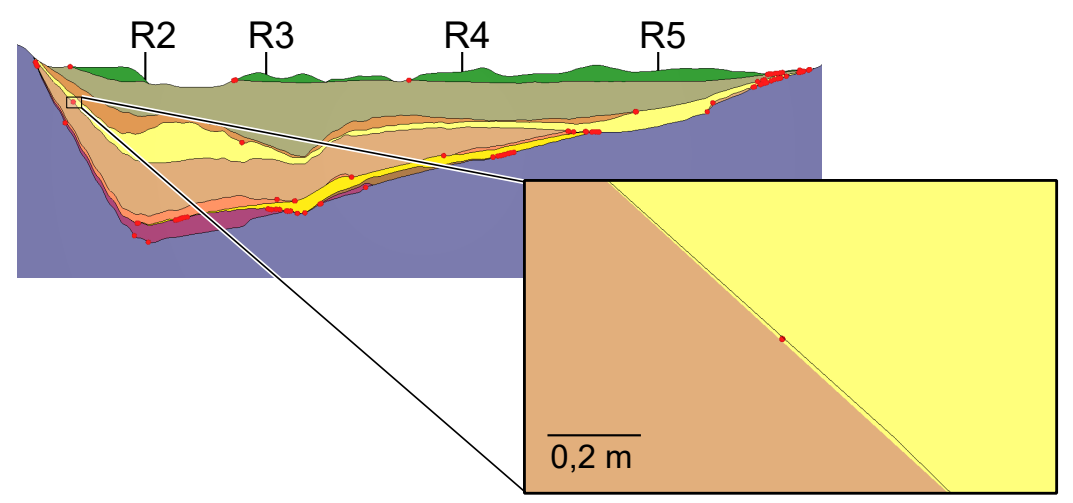

Figure 8. Consequence of the remeshing on the differences between the models M1 and M5. The differences are illustrated here by red dots. The outlines in black correspond to the interfaces in model M1; the colors correspond to the materials in model M5. Vertical exaggeration $=5$.
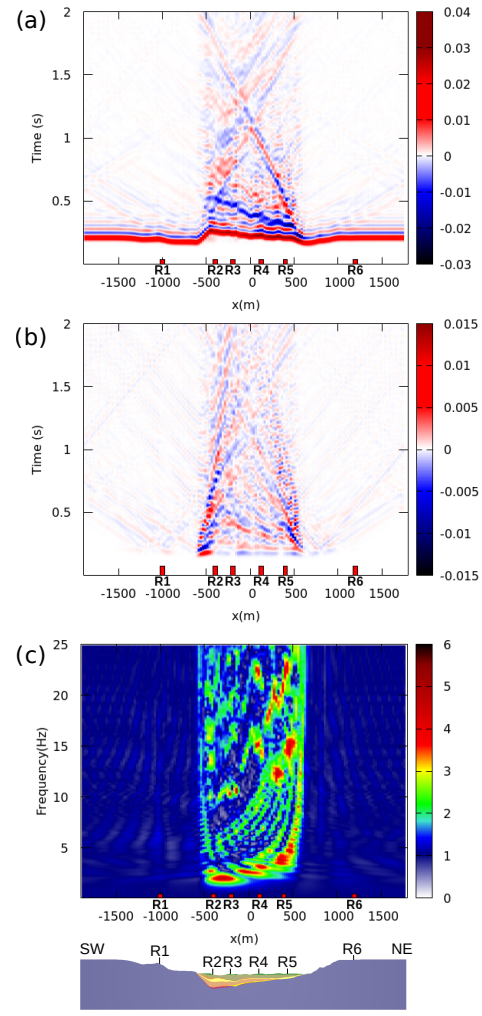

Figure 9. Vertical propagation of a shear plane wave in the reference model M1. Solutions at all surface receivers. The abscissa represents the position at the surface, the basin corresponding to the range $[-600 ; 700]$. (a) Time evolution of the horizontal velocity $V_{x}\left(\mathrm{~m} . \mathrm{s}^{-1}\right)$. (b) Time evolution of the vertical ground velocity $V_{z}$ $\left(\mathrm{m} . \mathrm{s}^{-1}\right)$. (c) Transfer functions of the horizontal ground velocity as a function of frequency. Spatial sampling: a receiver every 25 meters. Frequency sampling: $0.1 \mathrm{~Hz}$.

\subsection{Comparison of simulation results}

\subsubsection{Time evolution of horizontal and vertical components of ground motion}

In Figures ?? and ??, we present the error between the seismograms in M1 and in the various simplified models, for the two ground velocity components. These figures are con- structed simply by making the difference, at each receiver and for each time step, of the solutions in the two models that are compared. As previously for better visibility, we chose to show comparisons between models until 2s. For all the simplified models and the two velocity components, we notice that the main errors are related to the reflected waves inside the basin. For $V_{x}$, the highest errors (observed in models M2, M3 and M4) are localized at the basin edges, especially at the North-East end (around $x=500$ ), which corresponds to the areas most modified during simplifications (Figure ??). However, smaller but significant errors are present on the entire basin surface.

The analysis of the errors with respect to the reference model M1 makes it possible to separate the models into two groups, the first composed of models M2, M3 and M4, and the second containing M5 and M6. These two groups correspond to the different simplification strategies (Section ??). The maximum error on the horizontal component of the ground velocity fields is about $2.0 \times 10^{-3} \mathrm{~m} . \mathrm{s}^{-1}$ for the models in the first group, and lower than $8.4 \times 10^{-4} \mathrm{~m} . \mathrm{s}^{-1}$ for the models in the second group (Table ??). Note that the $\mathrm{PGV}$ of the horizontal component at the surface is $3.3 \times 10^{-2} \mathrm{~m} . \mathrm{s}^{-1}$. The maximum error on the vertical component varies between $2.0 \times 10^{-3}$ and $2.8 \times 10^{-4} \mathrm{~m} . \mathrm{s}^{-1}$ depending on model group. Nevertheless, the error is approximately twice larger for models M2 to M4 than for models M5 and M6 (Table ??).

In Figures ?? and ??, we plot the time evolution of horizontal and vertical velocity components at the six receivers, obtained for models M3 and M6 in comparison to the reference M1. Models M3 and M6 are those for which the computation times are the shortest ( 4 hours and 6 minutes, and 5 hours and 26 minutes, respectively). We first observe that the three solutions are very close to each other. Second, the general behavior of the difference (blue curves) is the same for the two velocity components. Discrepancies are mainly visible not on the direct wave but further on the reflections. However, the difference is larger between M1 and M3 than between M1 and M6. When comparing M1 and M3, the highest errors are observed at the four receivers located on the basin surface (R2 to R5) while the comparison between M1 and M6 shows negligible errors for R2, R3 and R4. This proves that automatic and localized simplifications have, in this study, a low impact far from the modifications. 

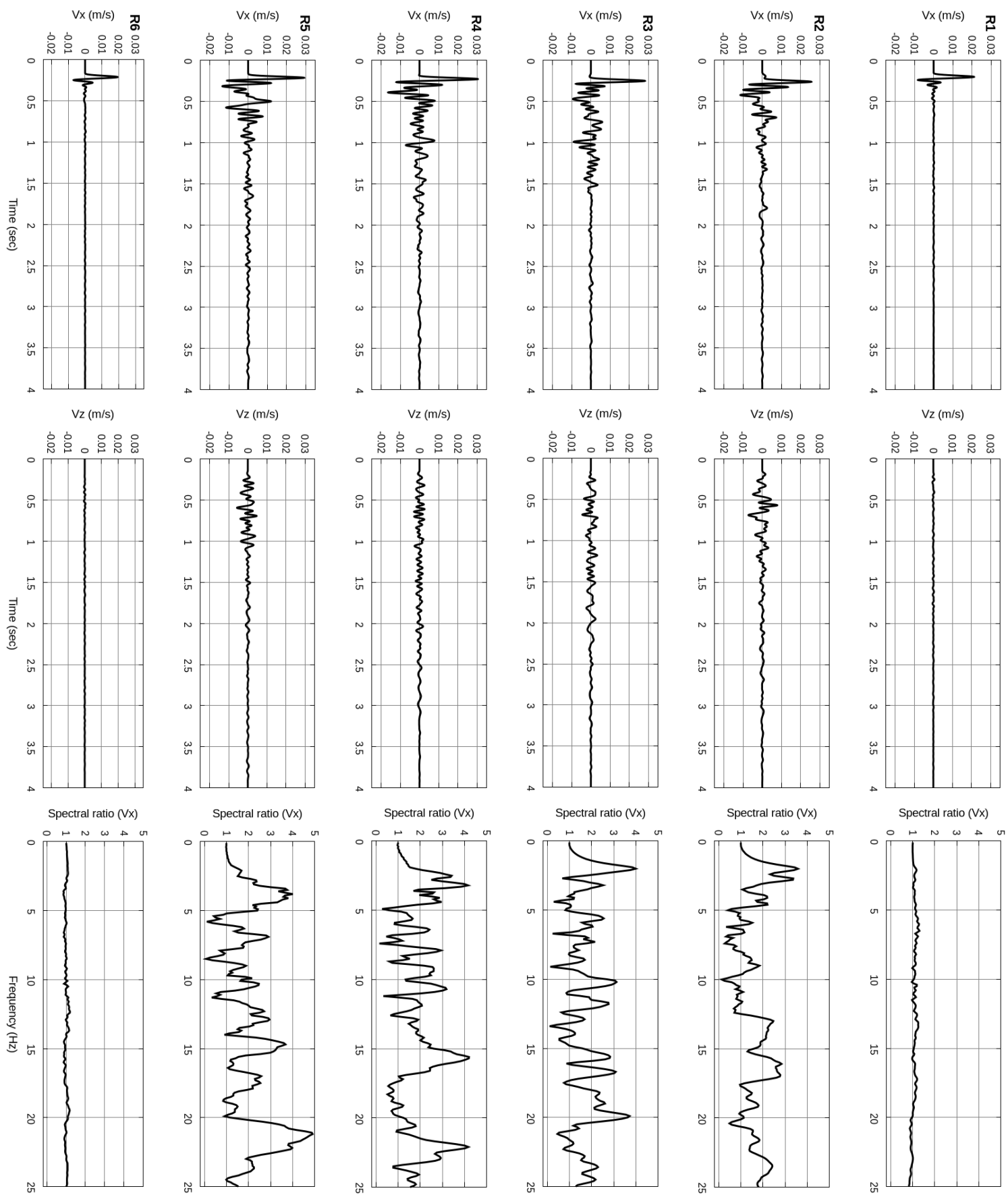

Figure 10. Vertical propagation of a shear plane wave in the reference model M1. Solutions at the six surface receivers R1 to R6. Left: Time evolution of the horizontal ground velocity $V_{x}\left(\mathrm{~m} . \mathrm{s}^{-1}\right)$. Center: Time evolution of the vertical ground velocity $V_{z}\left(\mathrm{~m} . \mathrm{s}^{-1}\right)$. Right: Transfer functions of the horizontal ground velocity as a function of frequency.

\subsubsection{Transfer functions}

In Figure ??, we present the errors on the transfer functions of the horizontal velocity at the surface between M1 and the simplified models. As for time solutions, this figure is constructed simply by making the difference, in each receiver and at each frequency step, of the solutions corresponding to the two models that are compared. No significant difference on the values of the fundamental frequencies and of the magnitude of highest amplifications is observed. We find the same groups of models (M2 to M4 on the one hand, and M5 and M6 on the other hand) as for ground velocities: differences on the transfer functions are also lower for models M5 and M6 than for models M2 to M4. The maximum error on transfer functions is about 0.4 for models M2 to M4, and 0.3 for models M5 and M6 (Table ??, upper part). These maximum error values are very close, but the differences are widely distributed for models M2 to M4 while they are concentrated around R5 and beyond $15 \mathrm{~Hz}$ for M5 and M6. This difference between models is clear when the error is calculated up to $15 \mathrm{~Hz}$ i.e., for frequencies corresponding to the 


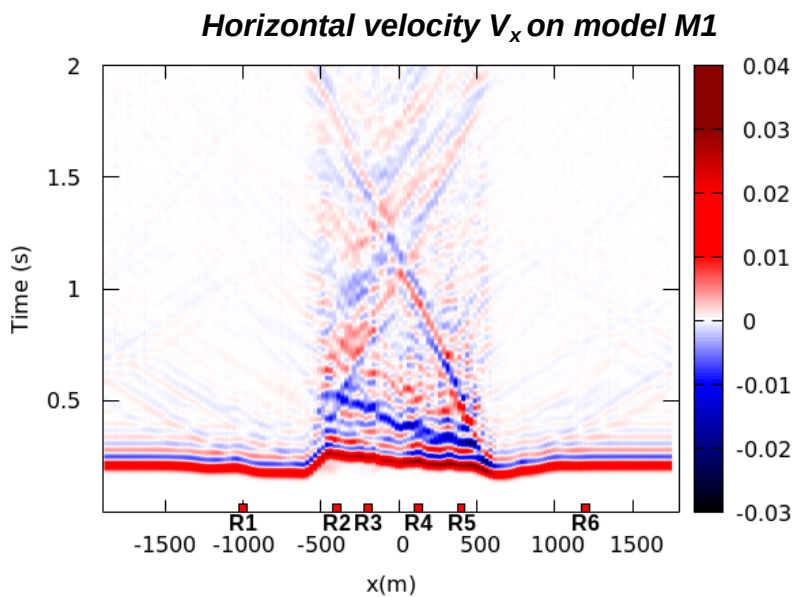

Difference between M2 and M1

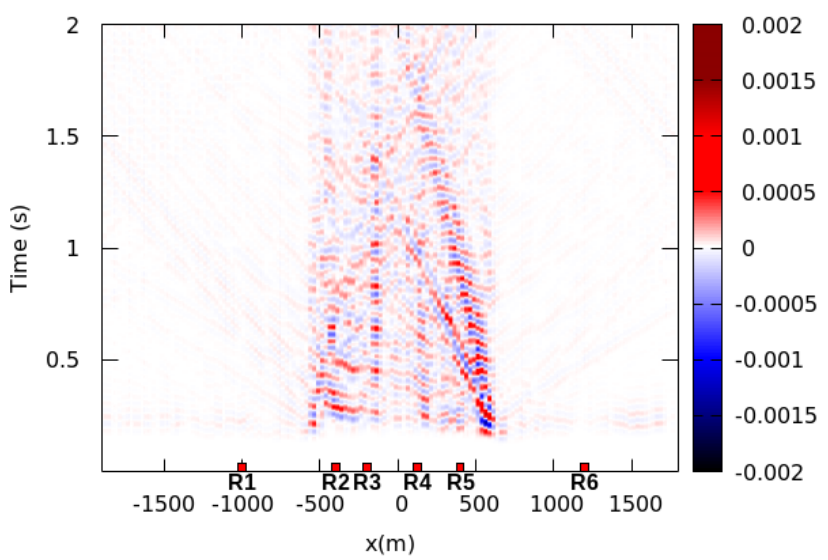

Difference between M3 and M1

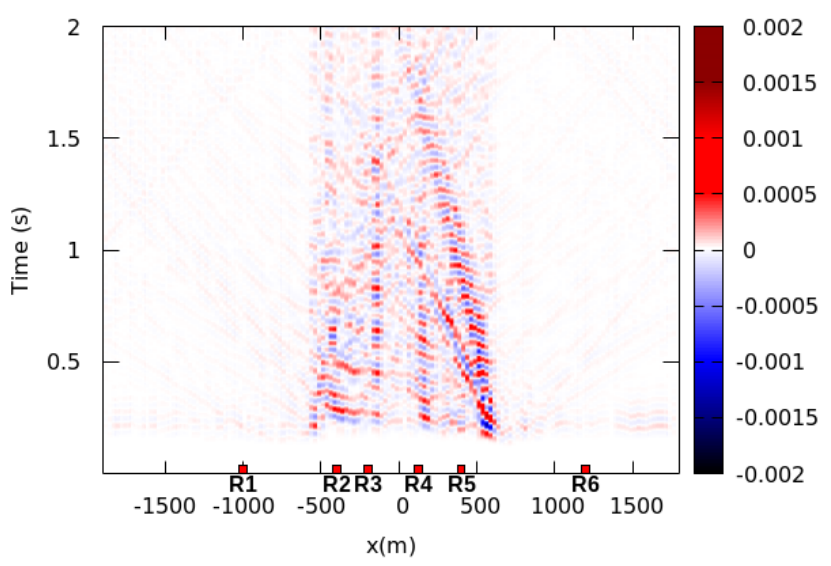

\section{Difference between M4 and M1}

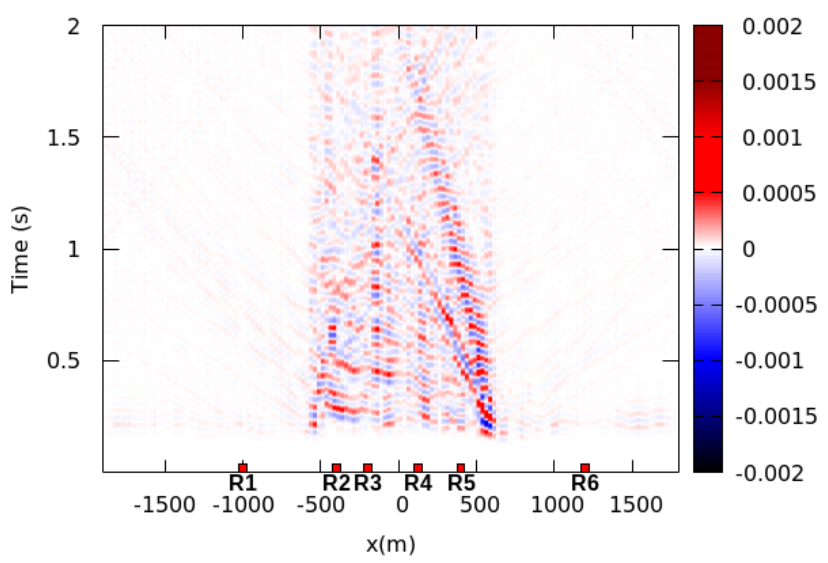

Difference between M5 and M1

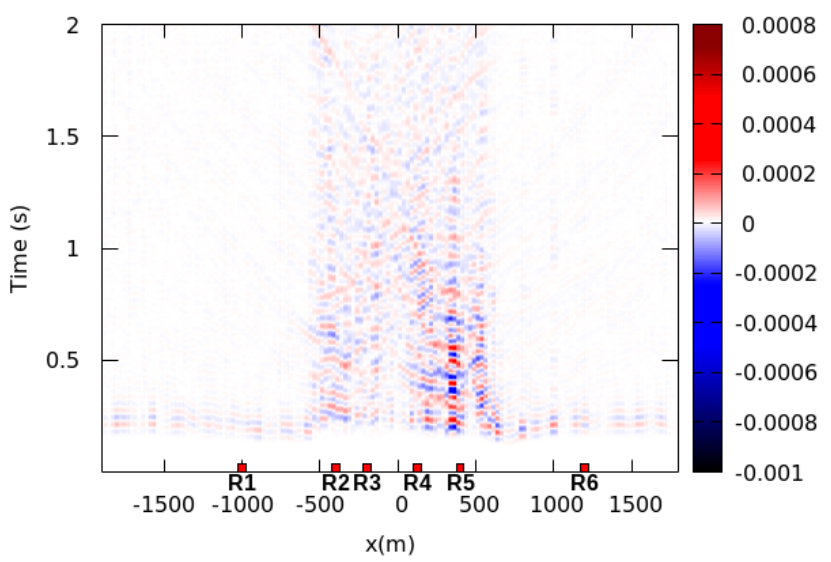

Difference between M6 and M1

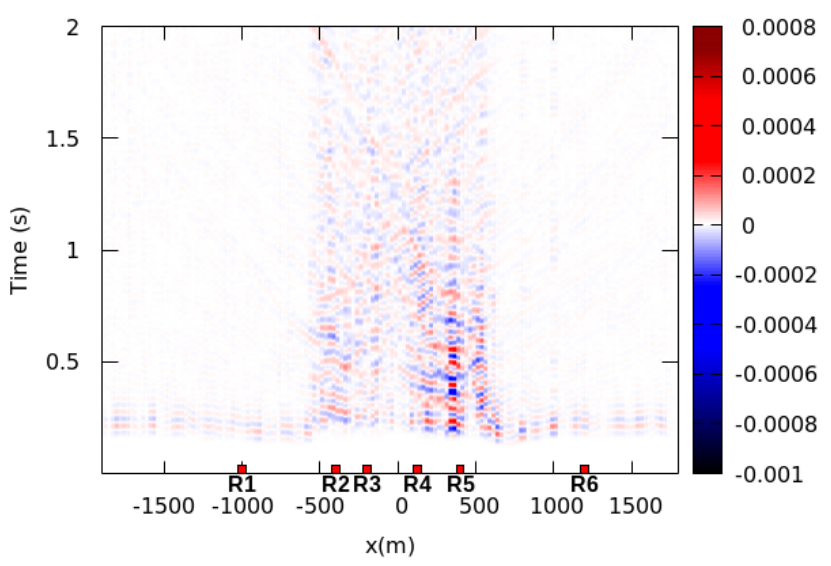

Figure 11. Differences of the horizontal velocity $V_{x}$ at the surface in M1 and in the five simplified models; the correspondence between the model numbers and the simplifications methods can be found in Table ??. The abscissa refers to the location along the profile, the ordinate is the time. For comparison with error levels, the maximum of $\left|V_{x}\right|$ for the model M1 is $3.31 \times 10^{-2} \mathrm{~m} . \mathrm{s}^{-1}$. Note that for visualization purposes, the color scales are adapted to extremum values and therefore differ according to the models. 
Table 4. Parameters of the time discretization and computation cost of the wave propagation simulations in models M1 to M6; the correspondence between the model numbers and the simplifications methods can be found in Table ??. $\Delta t$ is the time step. The number of iterations corresponds to a simulation until 4 seconds. All the simulations are performed on a classic desktop computer, with a Intel Core I7 2.70 GHz. The smallest height and the extension ratio are also reported.

\begin{tabular}{lcccccc}
\hline Model & M1 & M2 & M3 & M4 & M5 & M6 \\
\hline$\Delta t(\mathrm{~s})$ & $7.29 \times 10^{-7}$ & $7.63 \times 10^{-6}$ & $5.26 \times 10^{-5}$ & $1.45 \times 10^{-5}$ & $2.56 \times 10^{-5}$ & $4.00 \times 10^{-5}$ \\
Nb. iterations & 5480000 & 524000 & 76000 & 276000 & 156000 & 100000 \\
CPU time (s) & $1.08 \times 10^{6}$ & $1.01 \times 10^{5}$ & $1.48 \times 10^{4}$ & $5.28 \times 10^{5}$ & $3.03 \times 10^{4}$ & $1.96 \times 10^{4}$ \\
CPU time & $300 \mathrm{~h}$ & $28 \mathrm{~h} 10 \mathrm{~min}$ & $4 \mathrm{~h} 6 \mathrm{~min}$ & $14 \mathrm{~h} 46 \mathrm{~min}$ & $8 \mathrm{~h} 24 \mathrm{~min}$ & $5 \mathrm{~h} 26 \mathrm{~min}$ \\
CPU time ratio & 1 & 10.6 & 73.0 & 20.5 & 35.7 & 55.1 \\
\hline$H_{\text {min }}(\mathrm{m})$ & $2.77 \times 10^{-3}$ & $2.27 \times 10^{-2}$ & $1.76 \times 10^{-1}$ & $3.35 \times 10^{-2}$ & $8.71 \times 10^{-2}$ & $1.48 \times 10^{-1}$ \\
Extension ratio & 1 & 8.2 & 63.5 & 12.1 & 31.4 & 53.4 \\
\hline
\end{tabular}

flat part of the incident velocity spectrum (Table ??, lower part); the error value is divided by three for M5 and M6 compared to the case up to $25 \mathrm{~Hz}$. For the models M2 to M4, the relative error varies between 7.5 and $5.8 \%$, while it is only $2.2-2.3 \%$ for models M5 and M6. For all models, beyond $15 \mathrm{~Hz}$, the highest differences are found at high frequencies and low values of amplification; this artificially generates large percentages of relative error whereas the absolute error is rather low.

In Figure ??, we compare the transfer functions of the horizontal ground velocity, for the six receivers, obtained in models M3 and M6 and in the reference model M1. Again, the three solutions are very close one to another. As previously, the difference is also higher for M1 - M3 than for M1 - M6. In particular, differences between M3 and M1 are visible for frequencies higher than $2-3 \mathrm{~Hz}$ at receivers $\mathrm{R} 2$ to R5 while the error between M6 and M1 is negligible for all receivers at the basin surface up to $15 \mathrm{~Hz}$, which corresponds to the interval where the spectrum of the incident velocity is maximum. Automatic simplifications therefore have, in this study, a very small influence on the study of site effects.

\subsection{Additional case without attenuation}

To enhance and better see the effects of the geometric simplifications, we realized a second set of simulations considering that the medium is fully elastic. For this new series, we kept the same solver and exactly the same conditions as in the viscoelastic case simply by setting to zero the number of mechanisms of the GMB model; the system to be solved is then reduced to the linear elastic one. As previously, the final simulation time is 4 . $\mathrm{s}$; the reduction in computation time is around $15 \%$ compared to the previous case. In this section, we are mainly interested in the differences on the time solutions and the transfer functions between the model M1 and the other models. Concerning the figures, we limit ourselves to seismograms of the horizontal velocity and comparisons at receivers R4 and R5 which had the largest horizontal velocity amplification in the case with attenuation.

\subsubsection{Comparison of models on time solutions}

Figure ?? shows the error between the seismograms in M1 and in the various simplified models, for the horizontal ground velocity component. In this case, comparisons between models are shown over the duration of the simulation, i.e., until $4 \mathrm{~s}$. On both seismograms, the trapped waves inside the basin are clearly visible up to $4 \mathrm{~s}$. For all the simplified models, we observe the same trend as before but the differences are amplified by the absence of attenuation in the medium. The main errors are related to the reflected waves inside the basin; they are particularly visible for the first family of models (M2 to M4). For models M5 and M6, note that the color scales correspond to much lower values.

The maximum errors on both components of the ground velocity fields are approximately the double of those obtained in the case with attenuation. For the horizontal velocity component it is about $3.0 \times 10^{-3} \mathrm{~m} . \mathrm{s}^{-1}$ for the models M2 to M4, and $1.0 \times 10^{-3} \mathrm{~m} . \mathrm{s}^{-1}$ for the second group of models (Table ??). For the vertical component, the error is about $3.6 \times 10^{-3}$ for the first family of models and $5.6 \times 10^{-4} \mathrm{~m} . \mathrm{s}^{-1}$ for M5 and M6.

In Figure ??, the time evolution of horizontal velocity component is plotted at two receivers, $\mathrm{R} 4$ and $\mathrm{R} 5$, obtained for models M3 and M6 in comparison to the reference M1. The difference between models M3 and M6 is clear. Errors appear for the model M3 from the first reflections and maintain a similar amplitude throughout the simulation. On the other hand, they are of very low level for the model M6.

\subsubsection{Comparison of models on transfer functions}

In Figure ??, we present the transfer functions of the horizontal velocity at the surface and the associated errors between M1 and the simplified models. First of all on the transfer functions, we observe strong values (up to 16) of amplifications beyond $10 \mathrm{~Hz}$. Up to $15 \mathrm{~Hz}$, these values result from successive constructive waves trapped in the heterogeneous basin that have not been attenuated. Beyond $20 \mathrm{~Hz}$, strong amplifications can also come from the division by small values of the reference Fourier spectrum. The errors between models are visible for all models beyond $10 \mathrm{~Hz}$. We find the 
Table 5. Maximum errors $\left(m . s^{-1}\right)$ on horizontal and vertical velocity components at the surface. Correspondence between the model numbers and the simplifications methods can be found in Table ??.

\begin{tabular}{lccccc}
\hline Model & M2 & M3 & M4 & M5 & M6 \\
\hline Max. $V_{x}$ error & $1.86 \times 10^{-3}$ & $1.84 \times 10^{-3}$ & $1.80 \times 10^{-3}$ & $8.38 \times 10^{-4}$ & $8.36 \times 10^{-4}$ \\
Max. $V_{z}$ error & $2.06 \times 10^{-3}$ & $2.09 \times 10^{-3}$ & $1.98 \times 10^{-3}$ & $2.86 \times 10^{-4}$ & $2.85 \times 10^{-4}$ \\
\hline
\end{tabular}

same groups of models as previously: the level of error is twice as high for the first group compared to the second. The values of these errors are presented in the Table ??. By comparing models up to $25 \mathrm{~Hz}$, the error on the transfer functions is about 2 for models M2 to M4 and reduces to $8.0 \times 10^{-1}$ for models M5 and M6. This corresponds to more than $20 \%$ error for the first group of models and less than $10 \%$ for the second, at frequencies higher than $18 \mathrm{~Hz}$. When the errors are analyzed up to $15 \mathrm{~Hz}$, the error is reduced to $3-4 \%$ for the models M5 and M6, i.e., twice the value of the first set of simulations.

In Figure ??, the transfer functions of the horizontal ground velocity, for R4 and R5, obtained in models M3 and M6 and in the reference model M1 are compared. For this particular case, the error is only multiplied by two for a better visibility. The three solutions are close one to another but differences are visible between M3 and M1 for frequencies higher than $6 \mathrm{~Hz}$ while the error between M6 and M1 is negligible. This additional series of simulations without attenuation confirms that automatic simplifications produce solutions closer to the reference model with a significant reduction in computation time. 

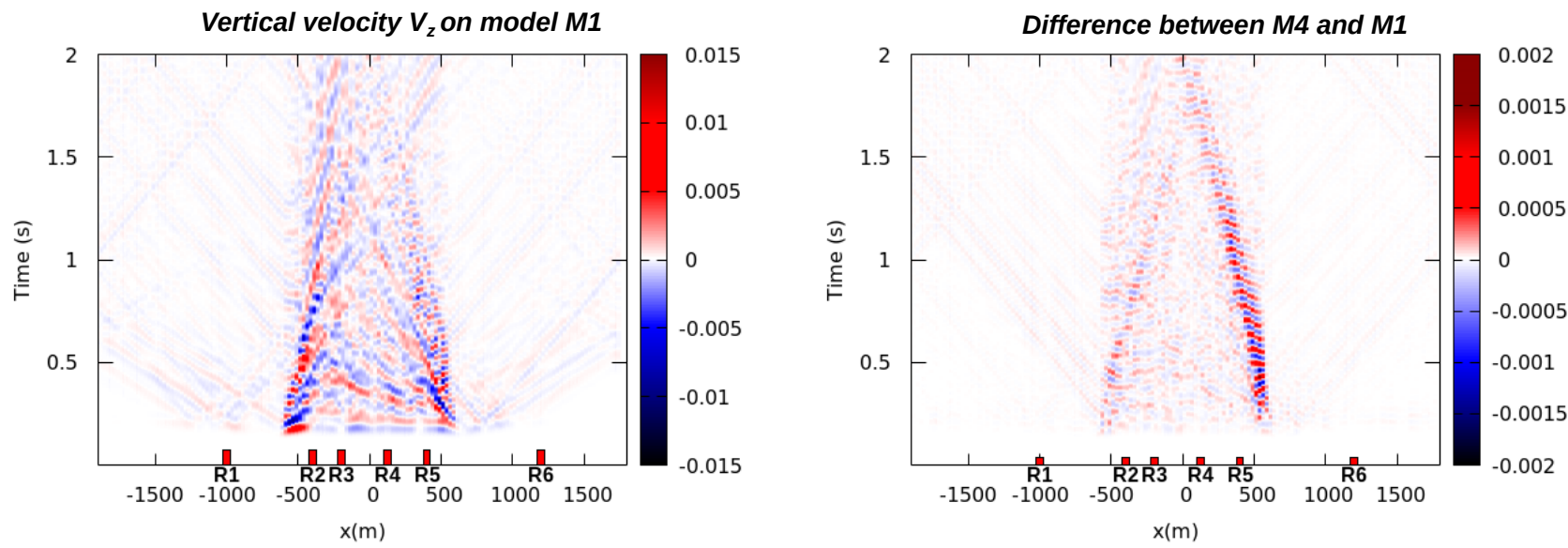

Difference between M2 and M1

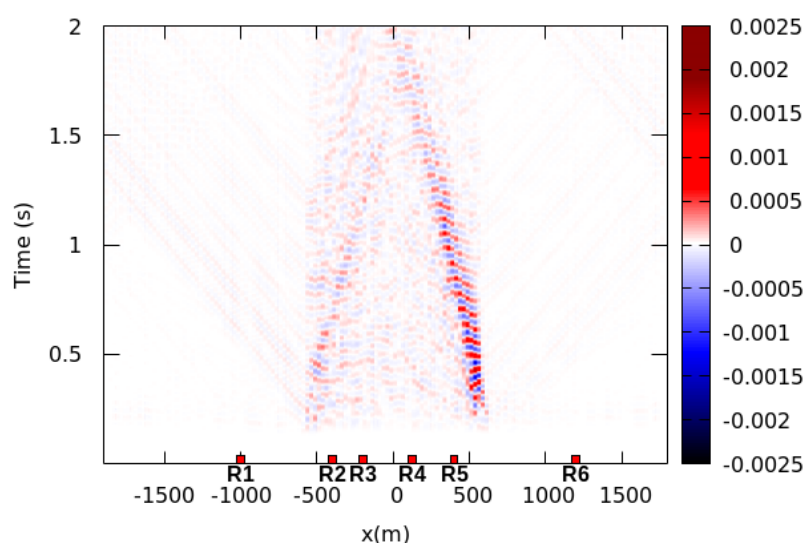

Difference between M5 and M1

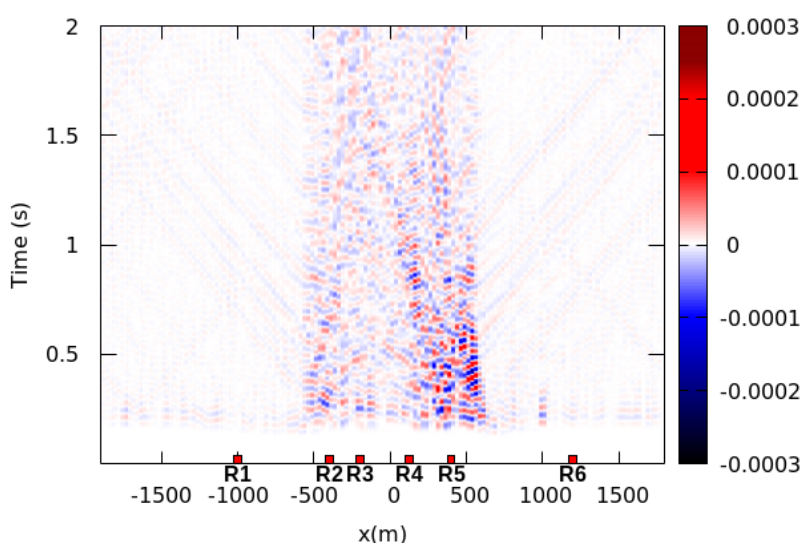

Difference between M3 and M1
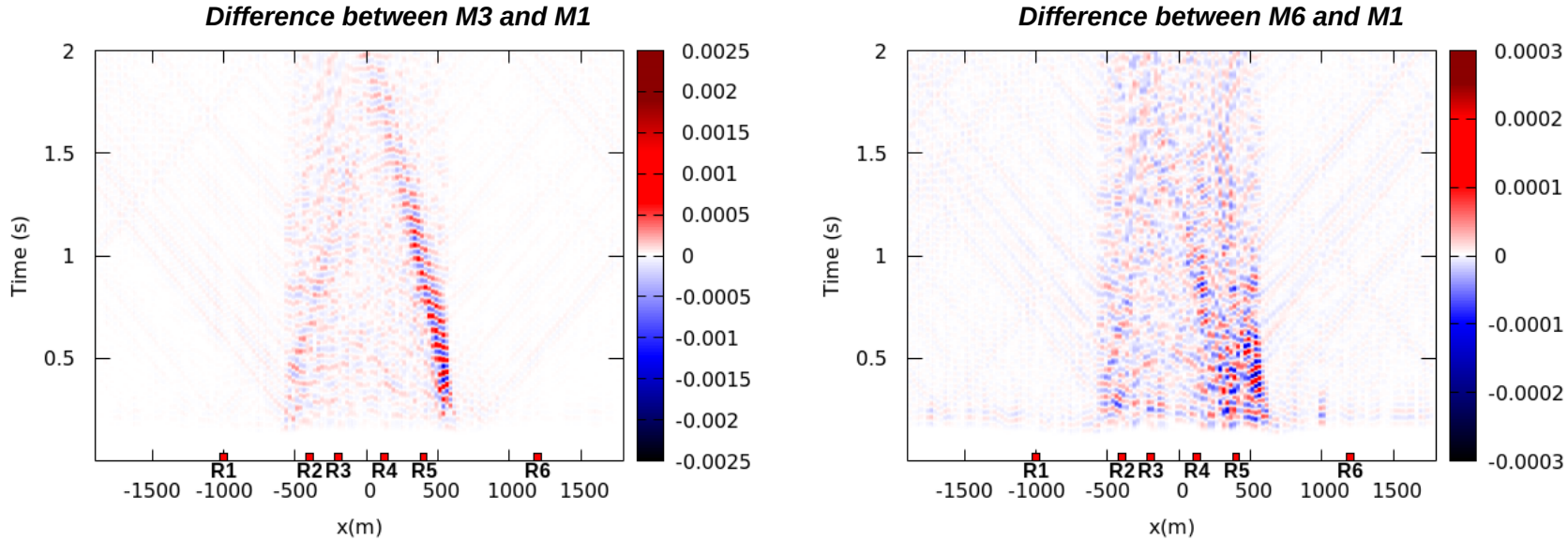

Figure 12. Differences of the vertical velocity $V_{z}$ at the surface in M1 and in the five simplified models; the correspondence between the model numbers and the simplifications methods can be found in Table ??. The abscissa refers to the location along the profile, the ordinate is the time. For comparison with error levels, the maximum of $\left|V_{z}\right|$ for the model M1 is $1.36 \times 10^{-2}$ m.s $s^{-1}$. Note that for visualization purposes, the color scales are adapted to extremum values and therefore differ according to the models. 
Comparison $M 1$ - M3
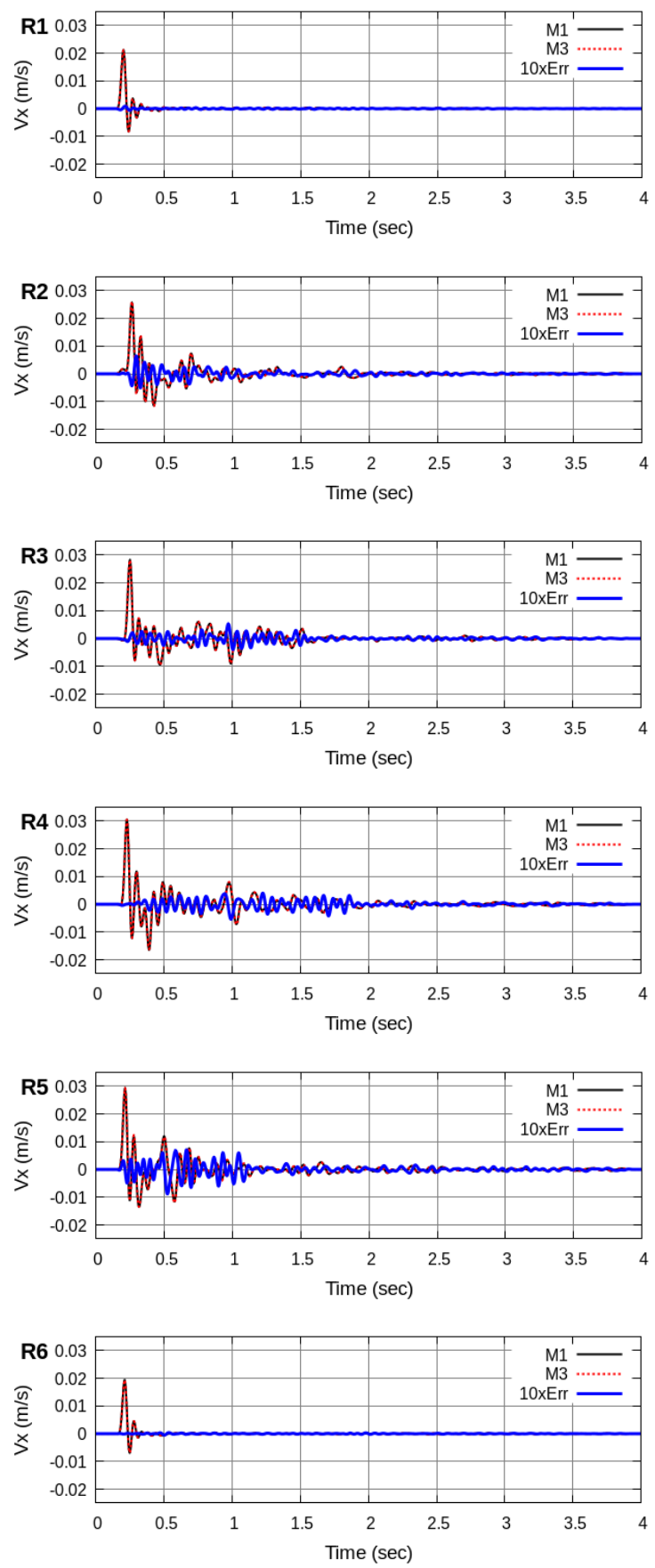

Comparison $M 1-M 6$
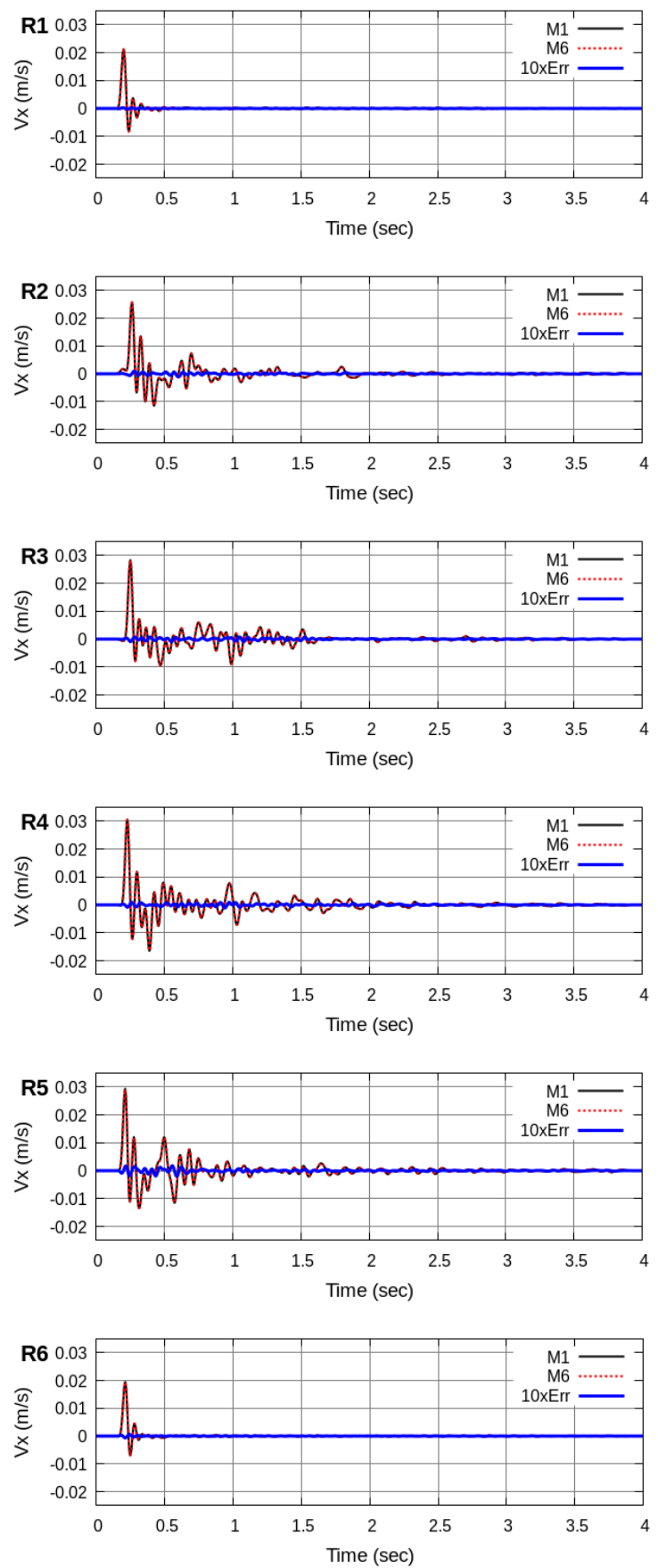

Figure 13. Time evolution of the horizontal velocity recorded at six surface receivers, comparison between models M1 and M3 (left) and M1 and M6 (right); the correspondence between the model numbers and the simplifications methods can be found in Table ??. The full black lines correspond to the reference M1, the red dashed lines to the simplified model. The difference between the two solutions, multiplied by a factor of ten, is in blue. 
Comparaison M1 - M3
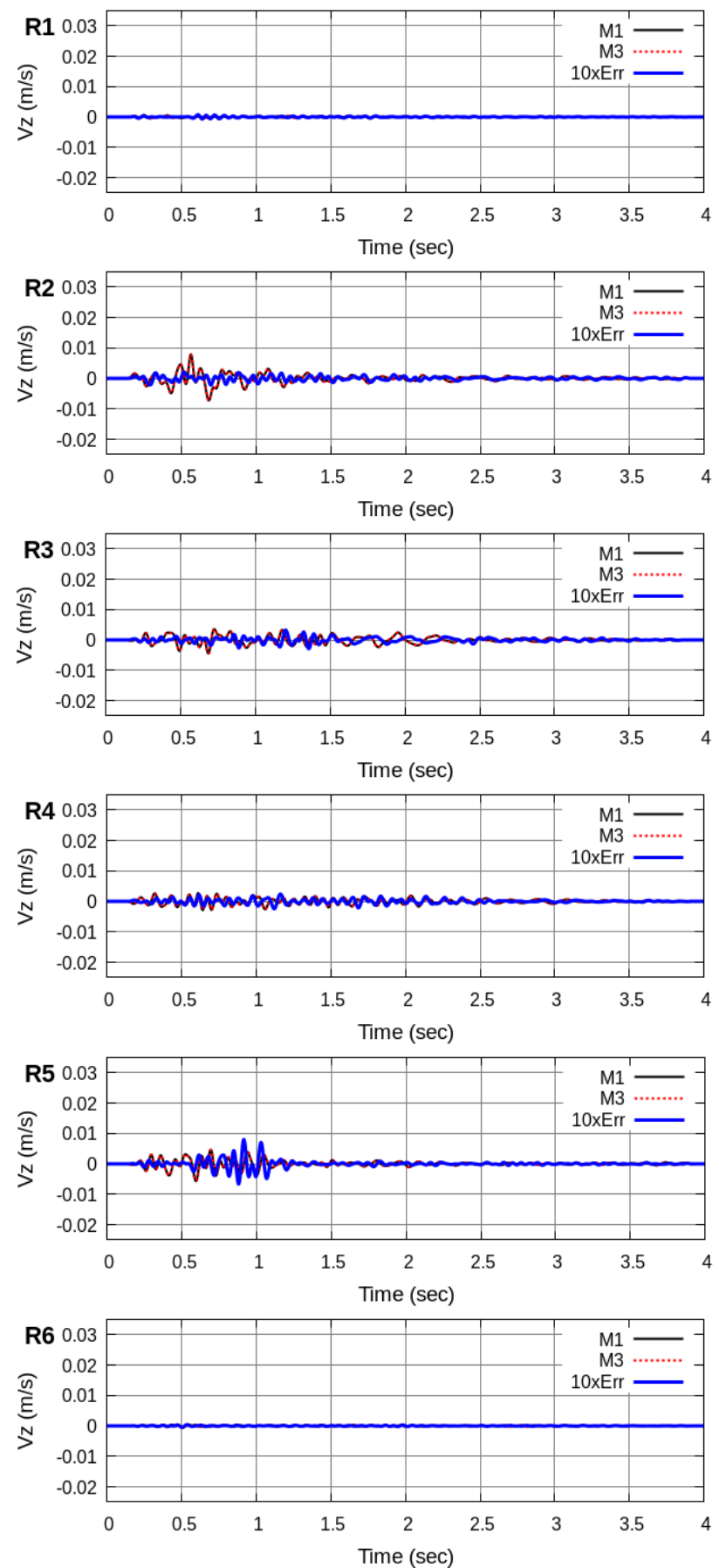

Comparaison M1 - M6
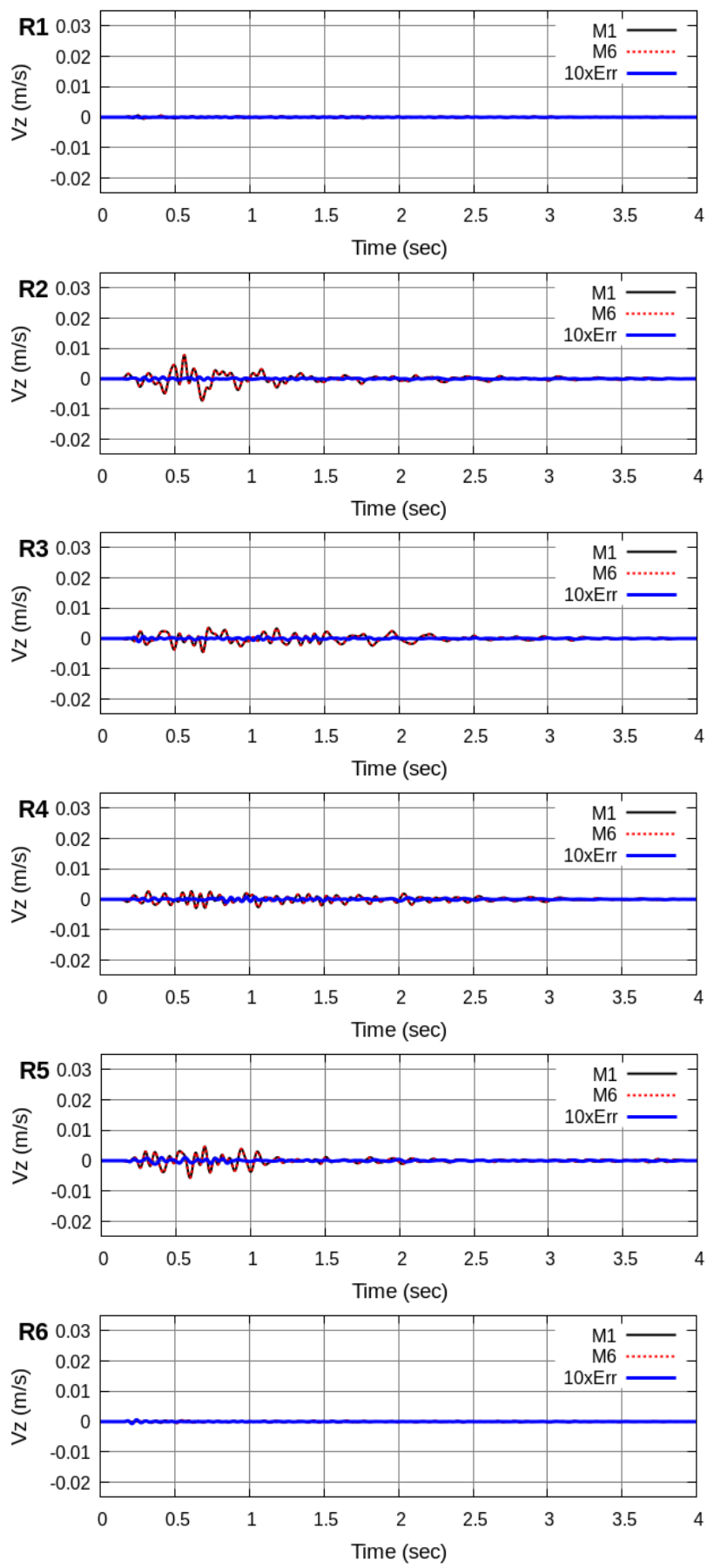

Figure 14. Time evolution of the vertical velocity recorded at six surface receivers, comparison between models M1 and M3 (left) and M1 and M6 (right); the correspondence between the model numbers and the simplifications methods can be found in Table ??. The full black lines correspond to the reference M1, the red dashed lines to the simplified model. The difference between the two solutions, multiplied by a factor of ten, is in blue. 
Wave amplifications on model M1
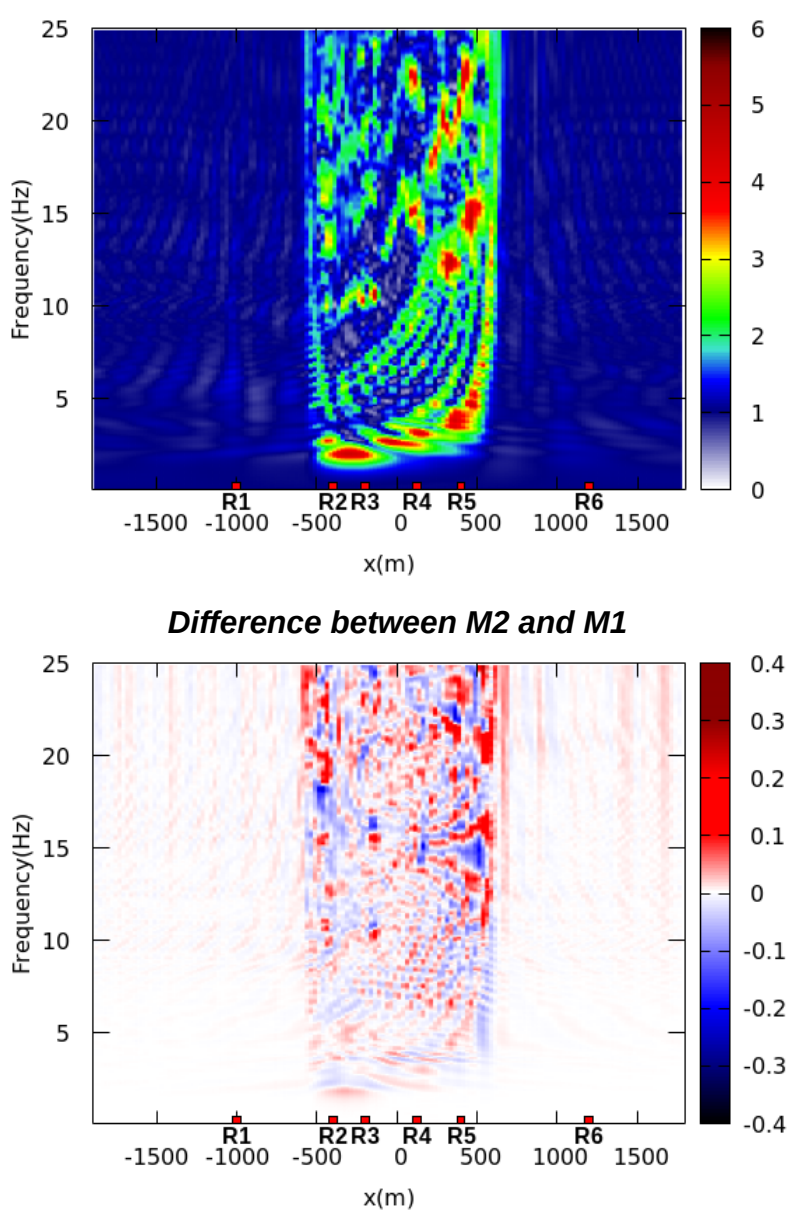

Difference between M3 and M1

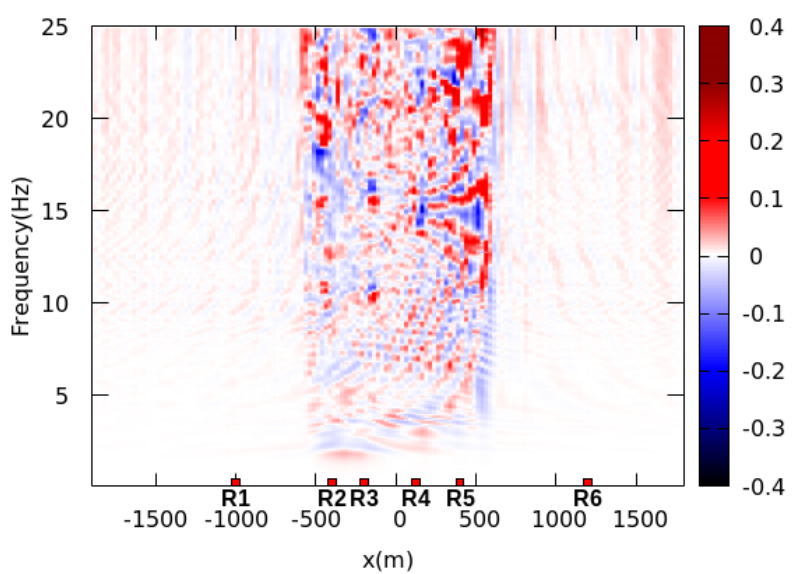

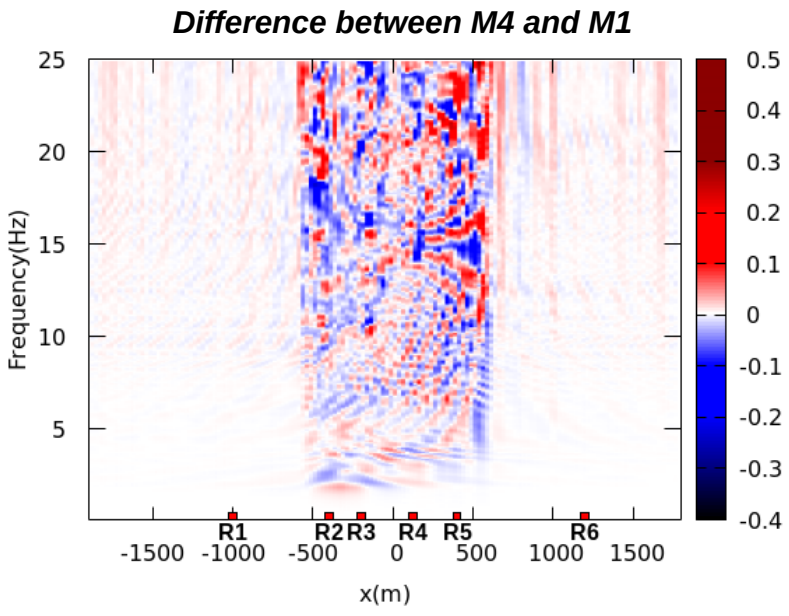

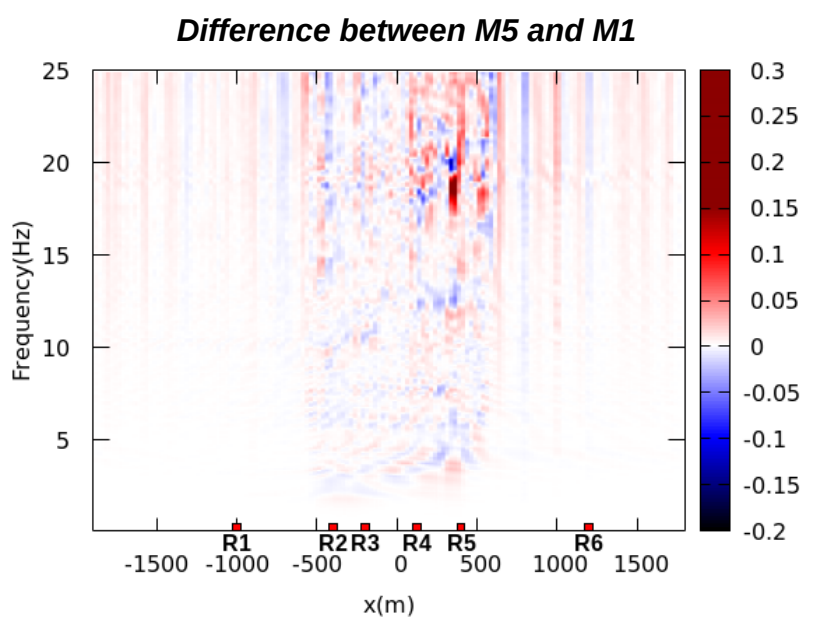

Difference between M6 and M1

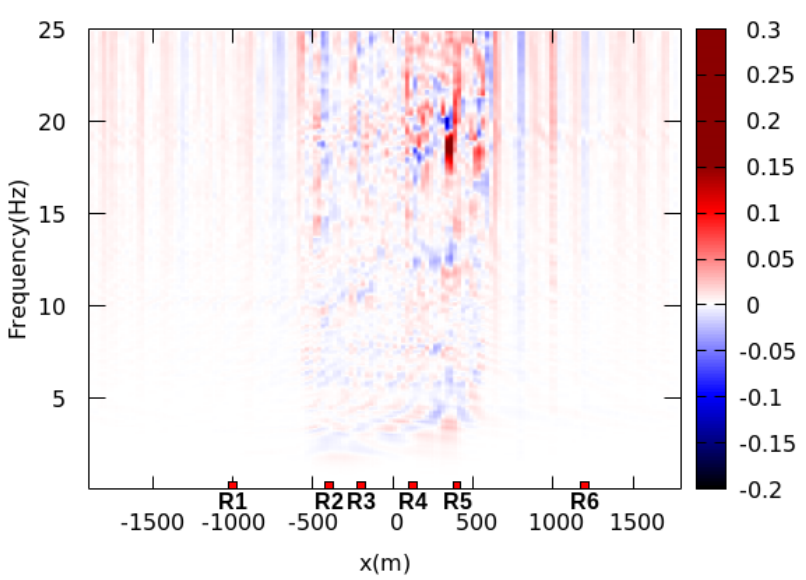

Figure 15. Differences between transfer functions of the horizontal velocity $V_{x}$ at the surface obtained between M1 and all simplified models; the correspondence between the model numbers and the simplifications methods can be found in Table ??. The abscissa corresponds to the location along the profile, the ordinate is the frequency. For comparison with error levels, the maximum amplification for the M1 model is 5.55. Note that for visualization purposes, the color scales are adapted to extremum values and therefore differ according to the models. 
Table 6. Maximum errors with respect to model M1 on the transfer functions of the horizontal ground velocity The correspondence between the model numbers and the simplifications methods can be found in Table ??. The location and the frequency corresponding to this maximum are given together with the relative error (in \%). The maximum amplification for the M1 model is 5.55 .

\begin{tabular}{lccccc} 
Model & M2 & M3 & M4 & M5 & M6 \\
\hline \multicolumn{5}{c}{ Errors on transfer functions up to $25 \mathrm{~Hz}$} \\
\hline Max. error & $3.89 \times 10^{-1}$ & $4.05 \times 10^{-1}$ & $4.14 \times 10^{-1}$ & $2.67 \times 10^{-1}$ & $2.68 \times 10^{-1}$ \\
Max. error (\%) & $17.1 \%$ & $17.8 \%$ & $18.7 \%$ & $8.2 \%$ & $8.3 \%$ \\
Location in $x(\mathrm{~m})$ & 550 & 550 & 550 & 325 & 325 \\
Frequency $(\mathrm{Hz})$ & 25.0 & 25.0 & 21.2 & 18.6 & 18.6 \\
\hline \multicolumn{5}{c}{ Errors on transfer functions up to $15 \mathrm{~Hz}$} \\
\hline Max. error & $2.62 \times 10^{-1}$ & $2.85 \times 10^{-1}$ & $2.72 \times 10^{-1}$ & $8.73 \times 10^{-2}$ & $8.65 \times 10^{-2}$ \\
Max. error $(\%)$ & $7.5 \%$ & $6.0 \%$ & $5.8 \%$ & $2.3 \%$ & $2.2 \%$ \\
Location in $x(\mathrm{~m})$ & 500 & 150 & 150 & 350 & 350 \\
Frequency $(\mathrm{Hz})$ & 15.0 & 14.4 & 14.4 & 12.6 & 12.6 \\
\hline
\end{tabular}

Table 7. Maximum errors $\left(m . s^{-1}\right)$ on horizontal and vertical velocity components at the surface. Case of a fully elastic medium. The correspondence between the model numbers and the simplifications methods can be found in Table ??.

\begin{tabular}{lccccc}
\hline Model & M2 & M3 & M4 & M5 & M6 \\
\hline Max. $V_{x}$ error & $3.03 \times 10^{-3}$ & $3.14 \times 10^{-3}$ & $3.03 \times 10^{-3}$ & $1.22 \times 10^{-4}$ & $1.22 \times 10^{-4}$ \\
Max. $V_{z}$ error & $3.64 \times 10^{-3}$ & $3.85 \times 10^{-3}$ & $3.74 \times 10^{-3}$ & $5.83 \times 10^{-4}$ & $5.65 \times 10^{-4}$ \\
\hline
\end{tabular}

Table 8. Maximum errors with respect to model M1 on the transfer functions of the horizontal ground velocity for a fully elastic medium. The correspondence between the model numbers and the simplifications methods can be found in Table ??. The location and the frequency corresponding to this maximum are given together with the relative error (in \%). The maximum amplification for the model M1 is 15.06 .

\begin{tabular}{lccccc} 
Model & M2 & M3 & M4 & M5 & M6 \\
\hline \multicolumn{5}{c}{ Errors on transfer functions up to $25 \mathrm{~Hz}$} \\
\hline Max. error & 2.35 & 2.06 & 2.15 & $8.07 \times 10^{-1}$ & $7.34 \times 10^{-1}$ \\
Max. error (\%) & $24.9 \%$ & $21.9 \%$ & $22.9 \%$ & $9.5 \%$ & $8.6 \%$ \\
Location in $x(\mathrm{~m})$ & 375 & 375 & 375 & 350 & 350 \\
Frequency (Hz) & 19.6 & 19.6 & 19.6 & 17.9 & 17.9 \\
\hline
\end{tabular}

Errors on transfer functions up to $15 \mathrm{~Hz}$

\begin{tabular}{lccccc}
\hline Max. error & 1.34 & 1.66 & 1.39 & $1.66 \times 10^{-1}$ & $1.71 \times 10^{-1}$ \\
Max. error (\%) & $15.8 \%$ & $19.6 \%$ & $16.4 \%$ & $3.0 \%$ & $4.0 \%$ \\
Location in $x(\mathrm{~m})$ & 150 & 150 & 150 & 525 & 350 \\
Frequency $(\mathrm{Hz})$ & 14.1 & 14.1 & 14.1 & 14.3 & 12.3 \\
\hline
\end{tabular}


Comparison $M 1$ - M3
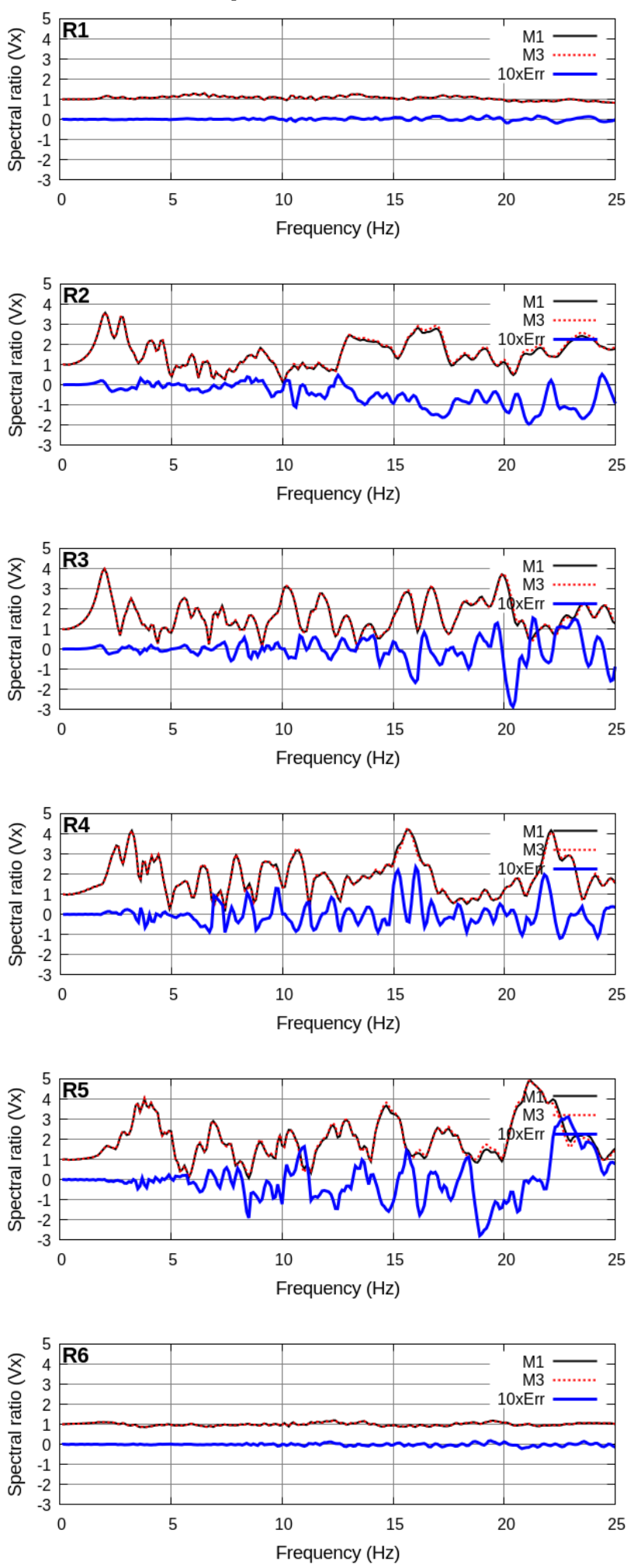

Comparison $M 1$ - M6
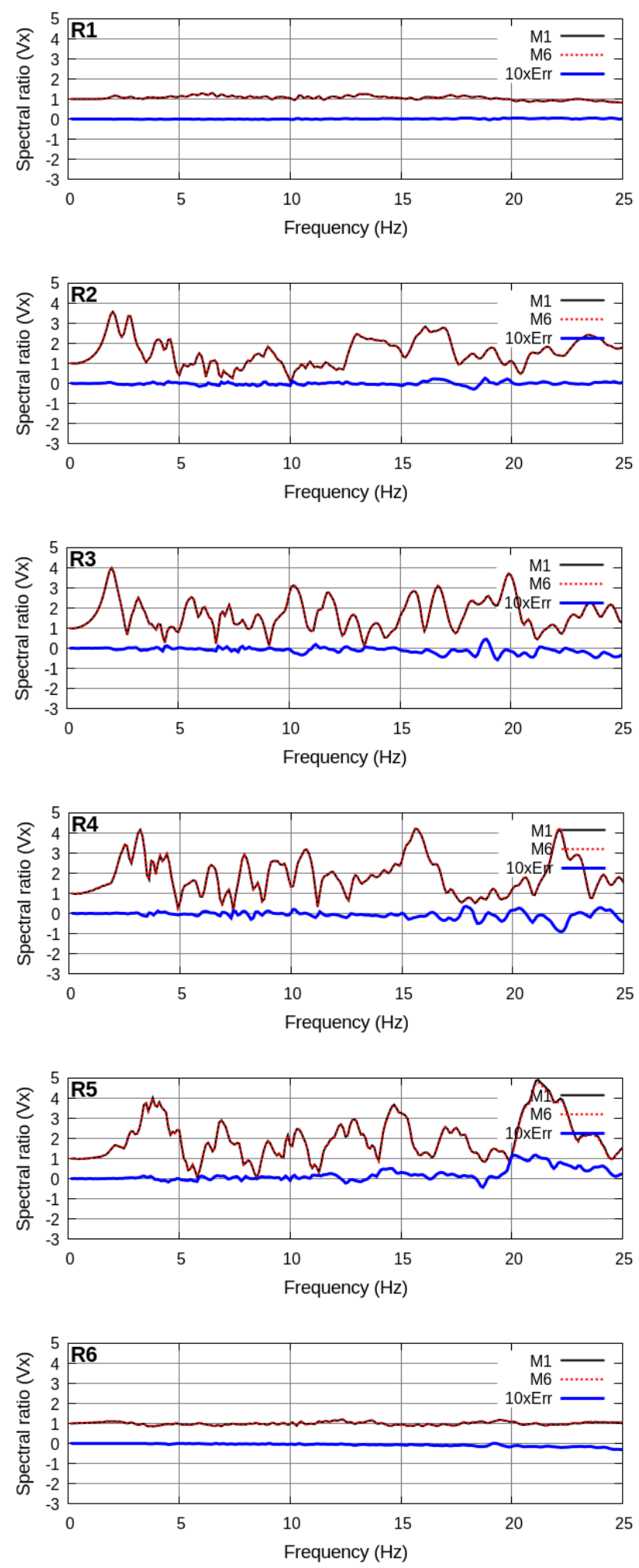

Figure 16. Transfer functions of the horizontal velocity recorded at six surface receivers, comparison between models M1 and M3 (left) and M1 and M6 (right); the correspondence between the model numbers and the simplifications methods can be found in Table ??. The full black lines correspond to the reference M1, the red dashed lines to the simplified model. The difference between the two solutions, multiplied by a factor of ten, is in blue. 


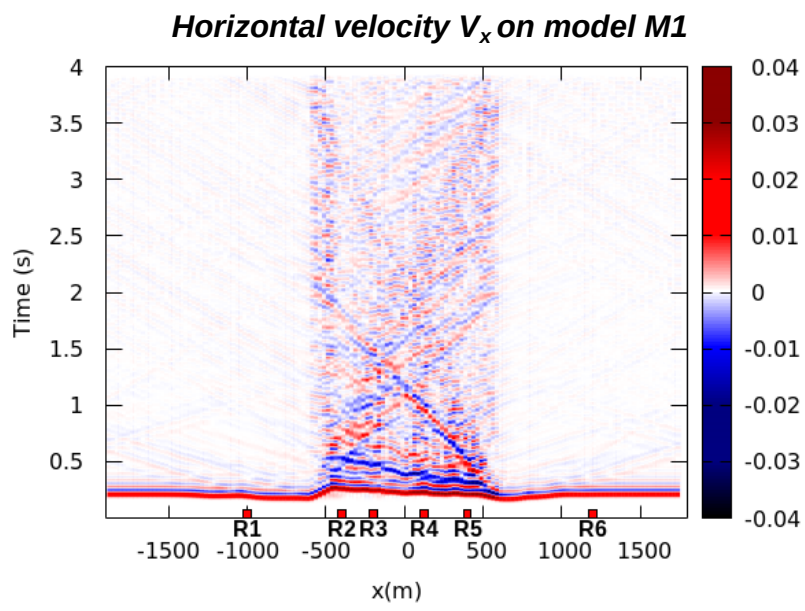

Difference between M2 and M1

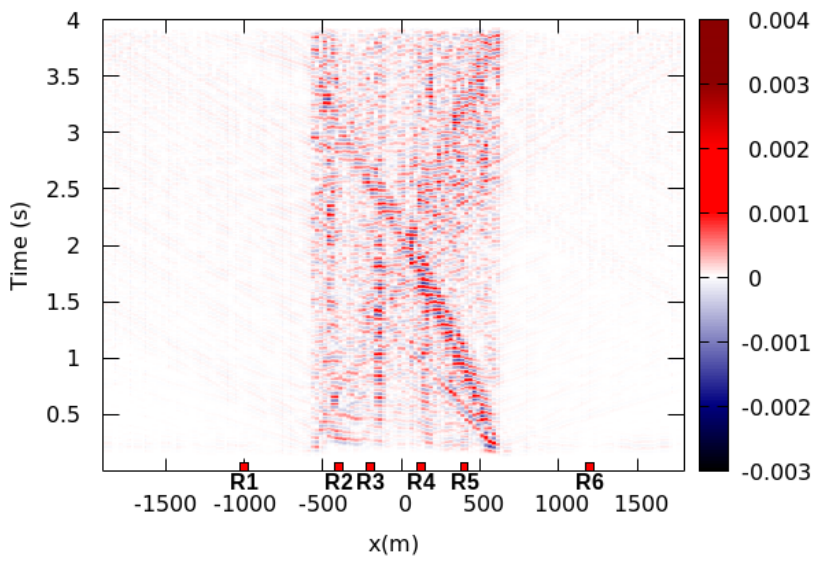

Difference between M3 and M1

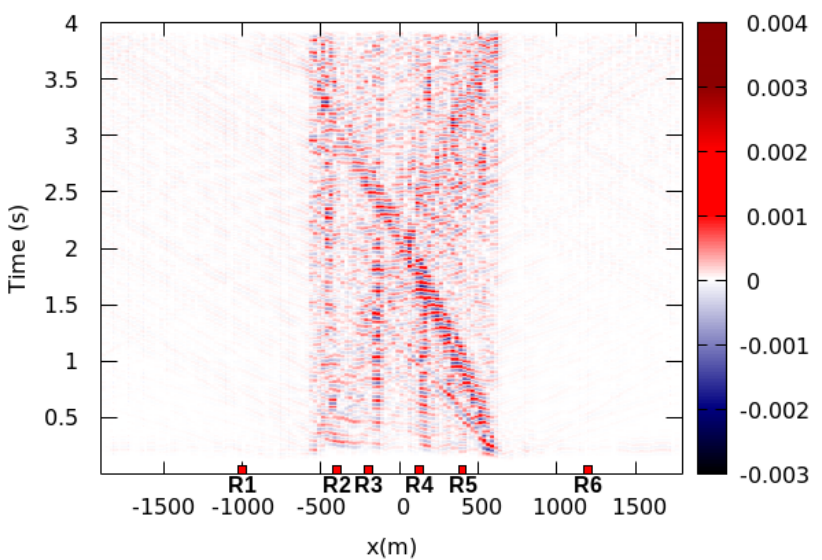

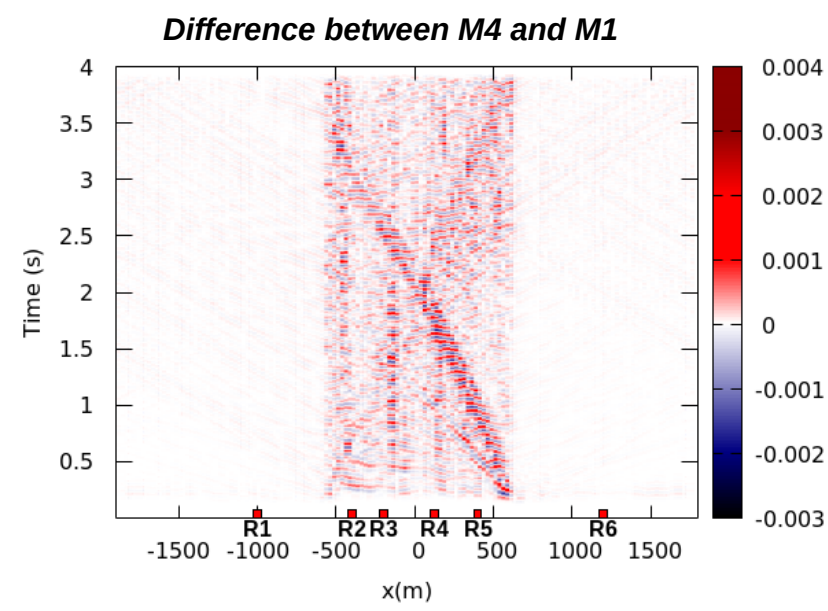

Difference between M5 and M1

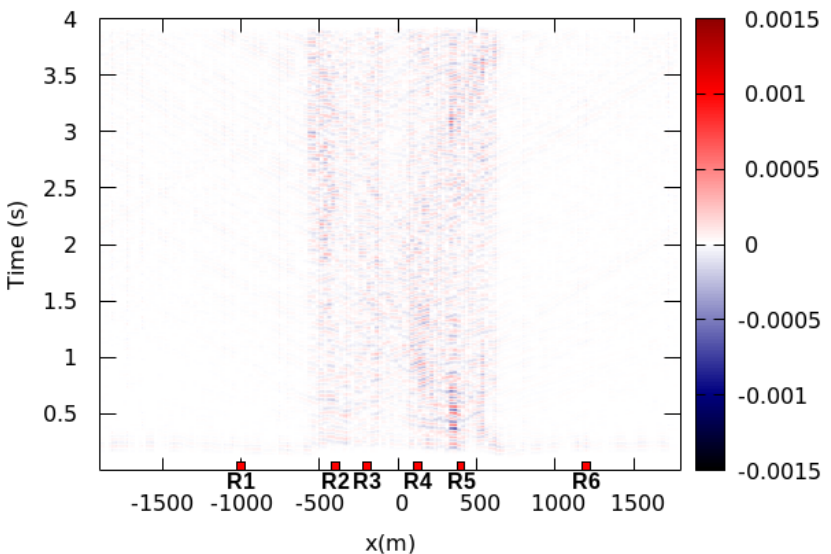

Difference between M6 and M1

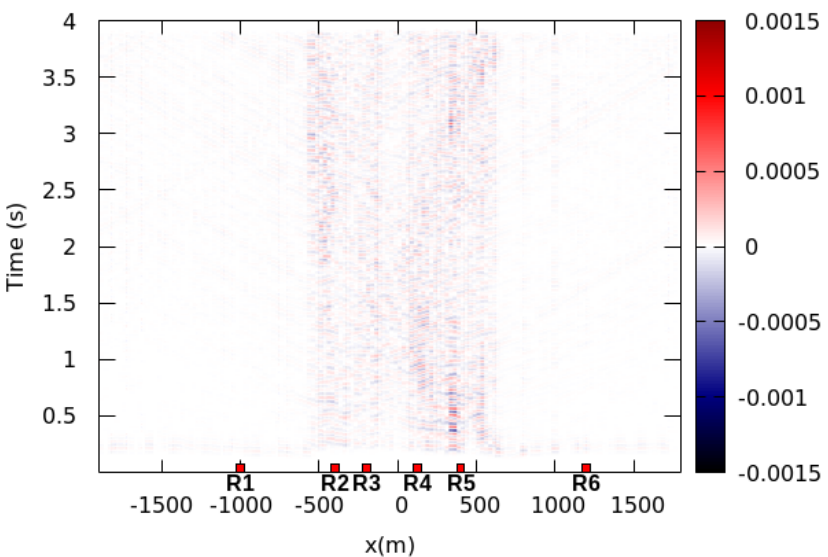

Figure 17. Differences of the horizontal velocity $V_{x}$ at the surface in M1 and in the five simplified models for a fully elastic medium. Correspondence between the model numbers and the simplifications methods can be found in Table ??. The abscissa refers to the location along the profile, the ordinate is the time. For comparison with error levels, the maximum of $\left|V_{x}\right|$ for the model M1 is $3.31 \times 10^{-2} \mathrm{~m} . \mathrm{s}^{-1}$. Note that for visualization purposes, the color scales are adapted to extremum values and therefore differ according to the models. 
Comparison M1 - M3
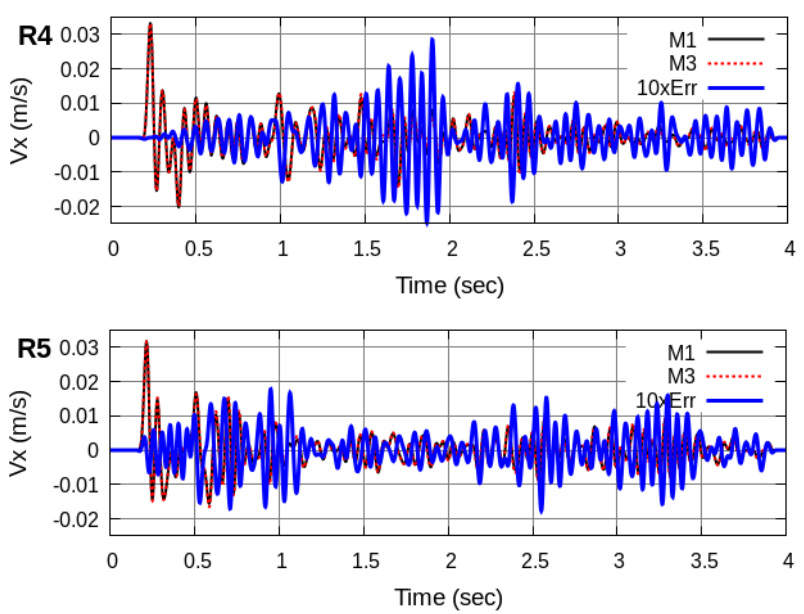

Comparison M1 - M6
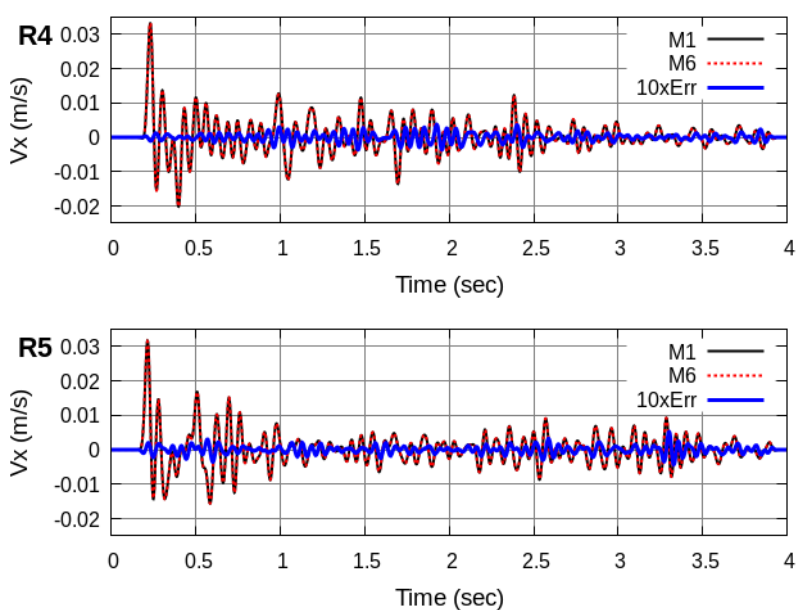

Figure 18. Time evolution of the horizontal velocity recorded at surface receivers R4 (top) and R5 (bottom), comparison between models M1 and M3 (left) and M1 and M6 (right) for a fully elastic medium. The full black lines correspond to the reference M1, the red dashed lines to the simplified model. The difference between the two solutions, multiplied by a factor of ten, is in blue. 


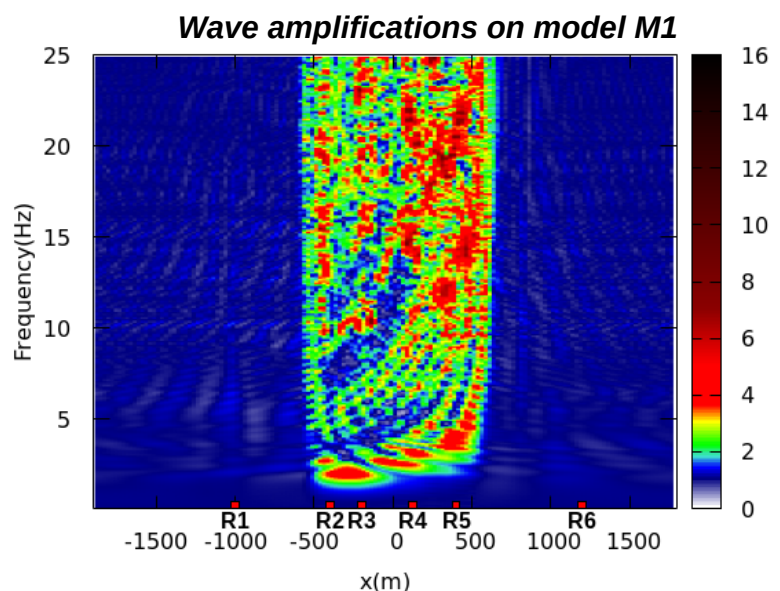

Difference between M2 and M1
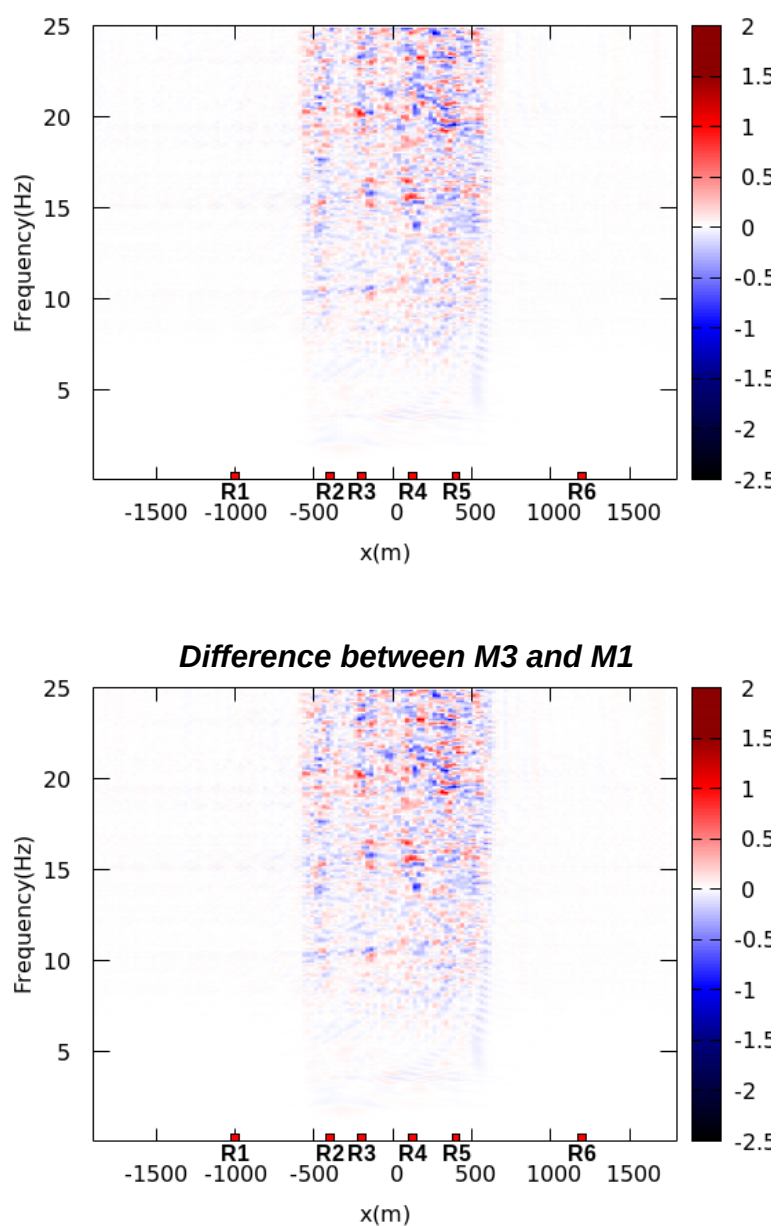

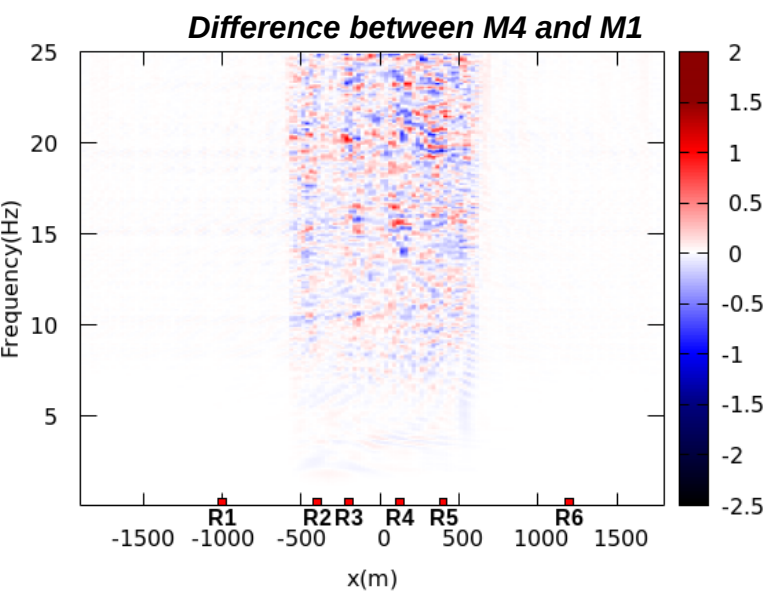

Difference between M5 and M1
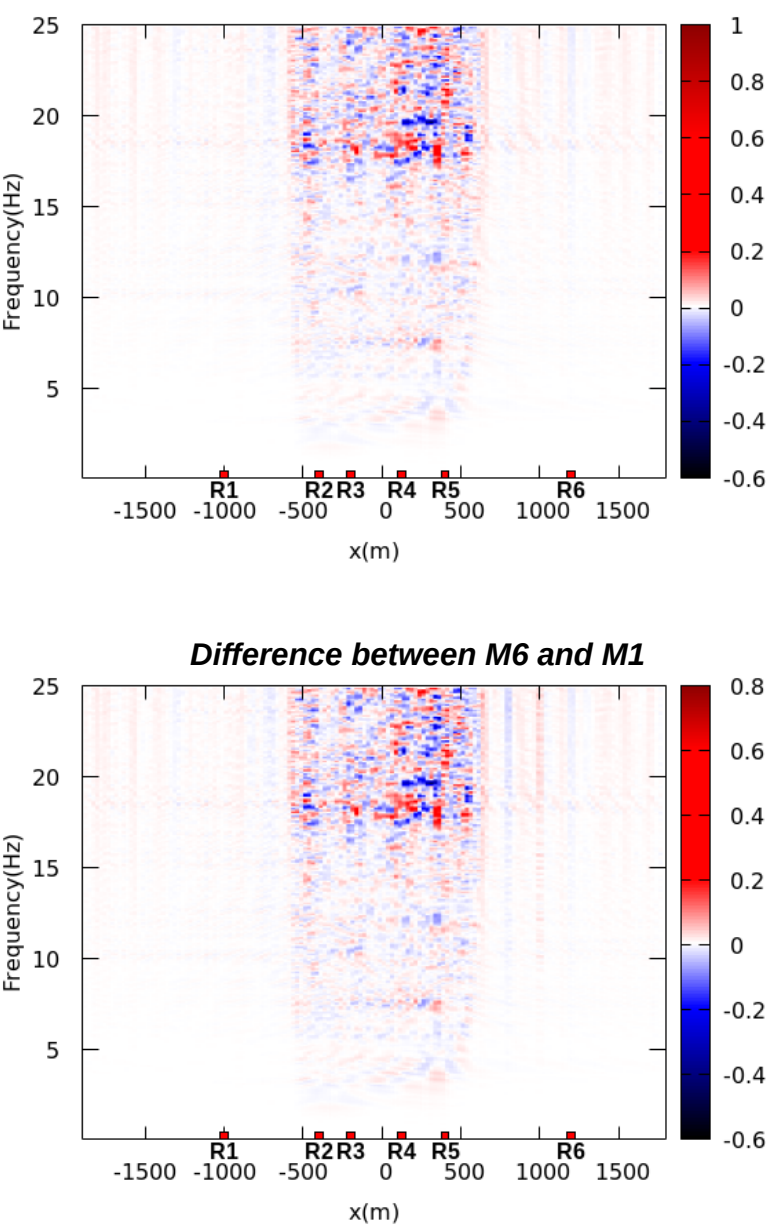

Figure 19. Differences between transfer functions of the horizontal velocity $V_{x}$ at the surface obtained between M1 and all simplified models for a fully elastic medium. Correspondence between the model numbers and the simplifications methods can be found in Table ??. The abscissa corresponds to the location along the profile, the ordinate is the frequency. For comparison with error levels, the maximum amplification for the M1 model is 15.06. Note that for visualization purposes, the color scales are adapted to extremum values and therefore differ according to the models. 
Comparison M1 - M3
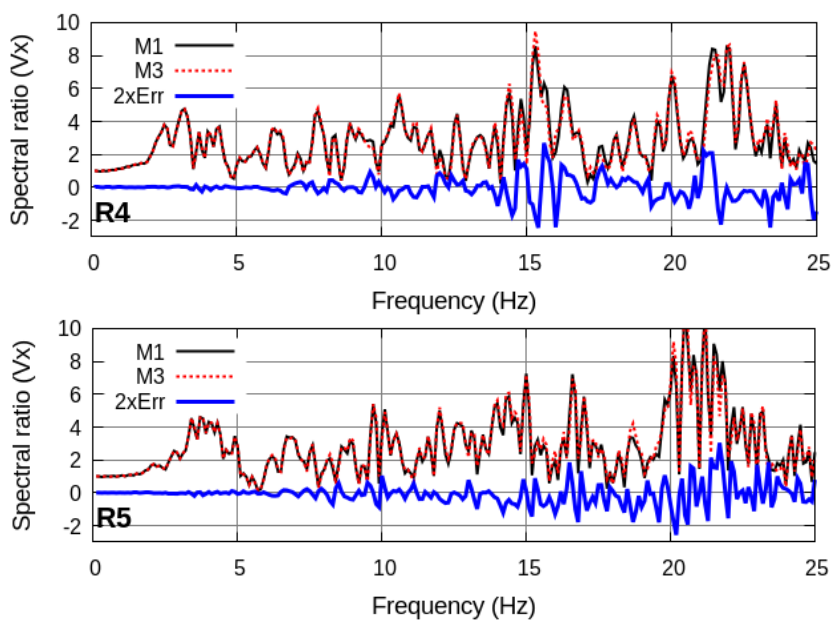

Comparison M1 - M6
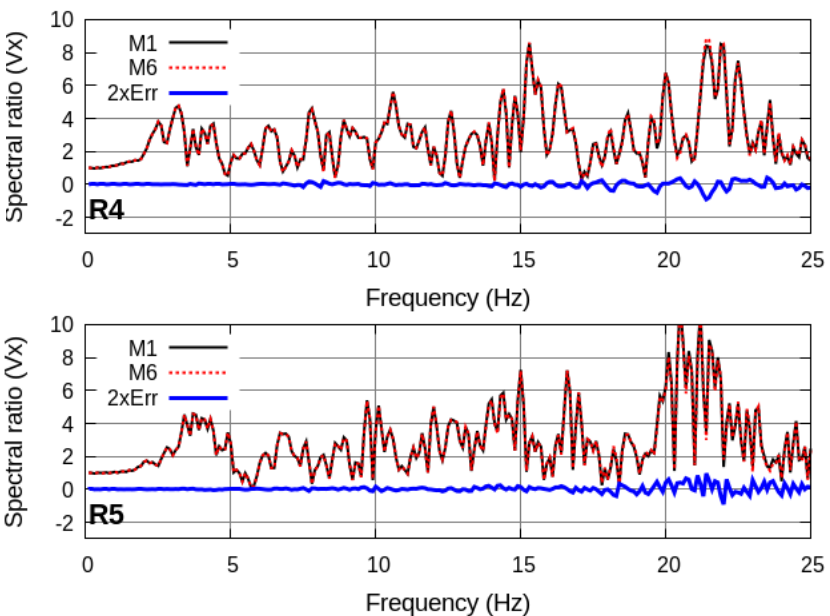

Figure 20. Transfer functions of the horizontal velocity recorded at surface receivers R4 (top) and R5 (bottom), comparison between models M1 and M3 (left) and M1 and M6 (right) for a fully elastic medium. correspondence between the model numbers and the simplifications methods can be found in Table ??. The full black lines correspond to the reference M1, the red dashed lines to the simplified model. The difference between the two solutions, multiplied by a factor of two, is in blue. 


\section{DISCUSSION}

Despite numerous experimental and numerical studies, $2 \mathrm{D} / 3 \mathrm{D}$ basin effects are often neglected in current engineering practices: building codes continue to estimate lithological site effects considering the vertical profile of shear wave velocity in $1 \mathrm{D}$ soil columns. The quantification of the overamplification of the ground motion compared to the $1 \mathrm{D}$ case can be included in these seismic codes through aggravation factors (???). One of the reason why $1 \mathrm{D}$ responses are still preferred in engineering practice is that $2 \mathrm{D} / 3 \mathrm{D}$ simulations in realistic geological models of sedimentary basins are computationally demanding. In this work, we have proposed a geometric simplification strategy to facilitate the application of $2 \mathrm{D}$ numerical wavefield simulations.

Some developments, based on modifications of the space and/or time discretization, aimed to solve the problem of small time steps induced by strongly heterogeneous models. The p-adaptivity, as mentioned in the introduction, allows an increase in the time step of small mesh elements by locally reducing the order of interpolation (e.g., ?). By coupling the solution of independent local problems with a global problem posed on a coarse mesh, the multiscale hybrid-mixed method (MHM) (?) handles high contrast interfaces on notaligned meshes. Other works relate to the approximation in time. For example, the use of local time-stepping techniques prevent the global time step from being the smallest value in the mesh (???). Coupled implicit-explicit time schemes (?) are freed from the stability criterion by applying an implicit scheme in the smallest elements without the disadvantages of a global implicit scheme.

As compared to these space and time modifications, our method is non intrusive in the sense that it does not require any modification of the numerical scheme or the seismic simulation code. However, it calls for choosing a priori a set of geometric parameters (the size of the exclusion zones and the minimal angle). This choice should be supported by physical and numerical reasoning, but looking for the best compromise between efficiency and accuracy cannot be achieved for every possible case study. To go further, it would be useful to consider not just the smallest triangle height, but the ratio between this height and the local P-wave velocity. Taking this velocity into account would imply local changes of the geometric parameters of the simplification method criteria, allowing it to focus on the areas most penalizing for the time step. As computation accuracy may suffer from large modifications, another perspective of this work would be to adapt the simplification criteria in space according to the contrasts of medium velocities inside the basin. We could also consider reducing differences in simulation results by adapting the magnitude of simplifications at strategic locations that may correspond to areas with a high influence on wave propagation. To do this, it would first be necessary to map these areas, by studying the wave paths between the sources and the receivers, using for example ray-based seismic illumination studies.

Overall, the above results show the interest of geometric model simplification methods to improve the mesh quality and thereby increase the time step and the efficiency of waveform simulation. For a negligible loss of accuracy (model M6), we observe a run time factor of 55 as compared to the run time on the reference model. Moreover, the best computational gain/accuracy ratio was observed on the model M6 obtained by a completely automatic simplification: this alleviates the scrutiny of several possible models by an expert and the subjectivity of manual simplification. In the presence of geological uncertainties, one could be tempted to use larger exclusion zones (and hence possibly allow for larger perturbations) of a geological interface in the areas where it is poorly constrained by observations (e.g., far from drill 
holes). This would, in our view, be an inappropriate interpretation of the parsimony principle. Indeed, the existence of structural uncertainties means that the true Earth may be different from the particular model used for the simulation. To approximate the nonlinear seismic response of the true Earth, detailed models may be needed. There is no reason whatsoever that the simplification approach used in this paper could systematically compensate for inaccuracies in the geological model. Instead, the simplification should only be considered as a computational convenience to accelerate the wavefield simulation. Therefore, in the presence of geological uncertainties, we strongly recommend to use stochastic structural modeling to sample possible structural models (?). Simplification should then be applied on each structural model realization to compute the wavefield sensitivity to geological uncertainties (?) or to implement Bayesian methods in an efficient way. In the Var valley case study, the model geometry is relatively well constrained both by borehole and seismic data (?), but such stochastic uncertainty studies could be envisioned in the future.

The simplification method we propose relies on the fact that slight geometric model changes have a negligible impact on the wavefield. This is usually the case in seismology, such as in our Var valley case-study. However, ?) show that a thin shallow layer below the free surface can have a strong impact on seismic waves, especially surface waves travelling over long distances, so that removing the layer or enlarging it as a whole would significantly change the waveforms. The magnitude of the allowed simplifications is extremely limited in this case, so our method would not be efficient. Beyond seismology, other wave phenomena would probably suffer from slight geometric modifications, such as the Helmholtz resonance in acoustics for instance (e.g., ?). In that case, a small change of the size of the resonator neck would considerably modify the resonance frequencies and band gaps.

In the study of site effects in sedimentary basins, comparison of simulations performed on a $2 \mathrm{D}$ cross-section with those using a $1 \mathrm{D}$ column shows the significant effect of $2 \mathrm{D}$ structures (?). We can reasonably extrapolate this observation to $3 \mathrm{D}$ models. To observe the effect of the threedimensional structures of basins on the ground motion, it would be helpful to run simulations on 3D models. This study shows that computation times can be extremely long on two-dimensional geological cross-sections without simplifications. In addition to the difficulty of generating a 3D mesh in geometrically complex areas, computation times for 3D simulations can impractically increase. Automatic 3D model simplifications, in combination with parallelization of physical solver schemes, would significantly reduce computation times. However, extensions of the proposed geometric simplification method to three-dimensional model are challenging to implement in a robust way, especially for building the model geometry (last step). We think that the multiplication of geometrical configurations in $3 \mathrm{D}$ would lead to an impractical number of case-by-case operations or to failure. To overcome this issue, we assume that an embedded geometrical approach as described in ?), constrained by a geological model topology analysis as operated here, is a possible way forward.

In complement to geometrical approximation techniques, homogenization could provide an interesting way forward to efficiently compute wavefields in complex geo- logical models (e.g., ?). Because it smooths heterogeneities smaller than the minimum wavelength to be propagated in the model, homogenization indeed prevents from handling both small and poorly shaped elements in wave simulations. In other words, the elements of the mesh of a homogenized medium are not constrained by the geological structures; they can be sized according to the wavelength, leading to an optimal computation cost in any wave simulator. Nonetheless, the homogenization process involves an elastostatic problem which can be solved by a classic finite element method (????). In this context, a mesh of the geological model is required, so the homogenization can take advantage of the automatic simplifications presented in this paper, even though the computation cost of a static finite element analysis is weakly sensitive to the size of the elements. Most of all, the homogenization theory is not yet able to provide an accurate solution (i.e., beyond the order 0 ) in the case of heterogeneities below a topographic surface. That is one of the reasons why homogenized media have not been used in seismic hazard assessment so far and why simulations in complex geomodels will remain a continuing need for long, asking for efficient simplification algorithms.

\section{CONCLUSIONS}

This work focuses on the influence of geometric simplifications of a $2 \mathrm{D}$ basin cross-section on wave propagation simulation in the context of a site effect study. The reference model corresponds to a $2 \mathrm{D}$ section of the basin of the lower Var valley, extracted from the 3D model of the basin recently proposed by ?). This section is discretized using a triangulated mesh in which an upgoing planar P-SV wave is propagated using a Discontinuous Galerkin method. Different simplification strategies (manual and automatic, modifying medium connectivity or not) are applied to generate five simplified versions of the reference model.

The results prove that the simplifications of the model significantly reduce the computational time: simulations on the simplified models become 10 to more than 70 times faster. Indeed, the geometrical simplifications introduced into the model improve the quality of the worst triangles, directly influencing the time step and consequently the computation time of the numerical simulations while preserving the stability of numerical method. The gain in computation time provided by the simplifications is considerable compared to the loss of accuracy unavoidably caused by these simplifications, both in the case of attenuating and purely elastic media. Similar site effects are indeed observed for all models: the simulations show the same amplified frequencies, the same level of amplification and lengthening of the signal duration in the basin, with a notable trend depending on the basin depth.

In detail, however, the results show that simplified models based on the automatic method introduced in ?) have two advantages: (1) this method automates the operations of simplifications, which allows the use of systematic and reproducible criteria based on geometric parameters, (2) the simplifications are applied cautiously, by only modifying the model locally at the level of geometrically complex zones. As a result, numerical wave propagation simulations performed on simplified models using this method are closer to the ref- 
erence results than those corresponding to models simplified manually or by merging media with similar physical properties. In addition, simulations are obtained in a significantly shorter time (between 5 and 8 hours against more than 12 days for the initial model) while automatic cross-section simplification takes only few seconds. The mesh simplifications therefore make it possible to carry out numerical simulations which are hardly feasible in the initial model.

\section{ACKNOWLEDGMENTS}

This work was performed in the frame of the RING project (http://ring.georessources.univ-lorraine.fr/) at Université de Lorraine. We would like to thank for their support the industrial and academic sponsors of the RING-GOCAD Consortium managed by ASGA. The automatic simplification methods are implemented in the SCAR software available to the RING Consortium members. This study was also supported by the French government, through the UCAJEDI Investments for the Future project managed by the National Research Agency (ANR) with the reference number ANR-15-IDEX-01. The authors would also like to thank Etienne Bertrand, Diego Mercerat and Ophélie Rohmer for fruitful discussions and for building and providing the 2D data used in this study, and Jeanne Pellerin and Bruno Lévy for discussions on the simplification strategy. Finally, we thank Y. Capdeville, an anonymous reviewer and Editor V.M. Cruz-Atienza for constructive and insightful comments which contributed to improving this paper.

\section{Data availability}

The software and the meshes underlying this article are freely available on RING-team GitHub repository at https: //github.com/ring-team/SCAROpen

\section{Appendix A}

This appendix shortly presents the $2 \mathrm{D}$ cross-section repair and simplification algorithm used in this article for generating models M3, M5 and M6. Its aims is to remain short; for more details the reader is referred to ?) and for complete details to ?) and GitHub repository (see ??).

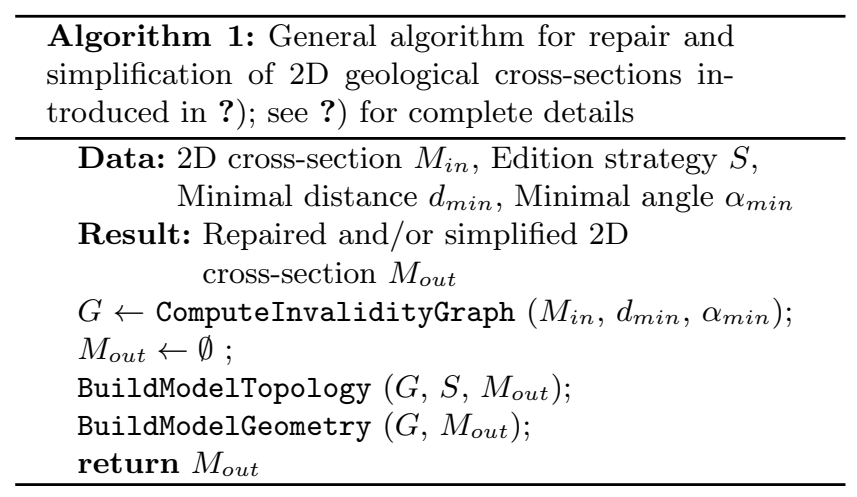

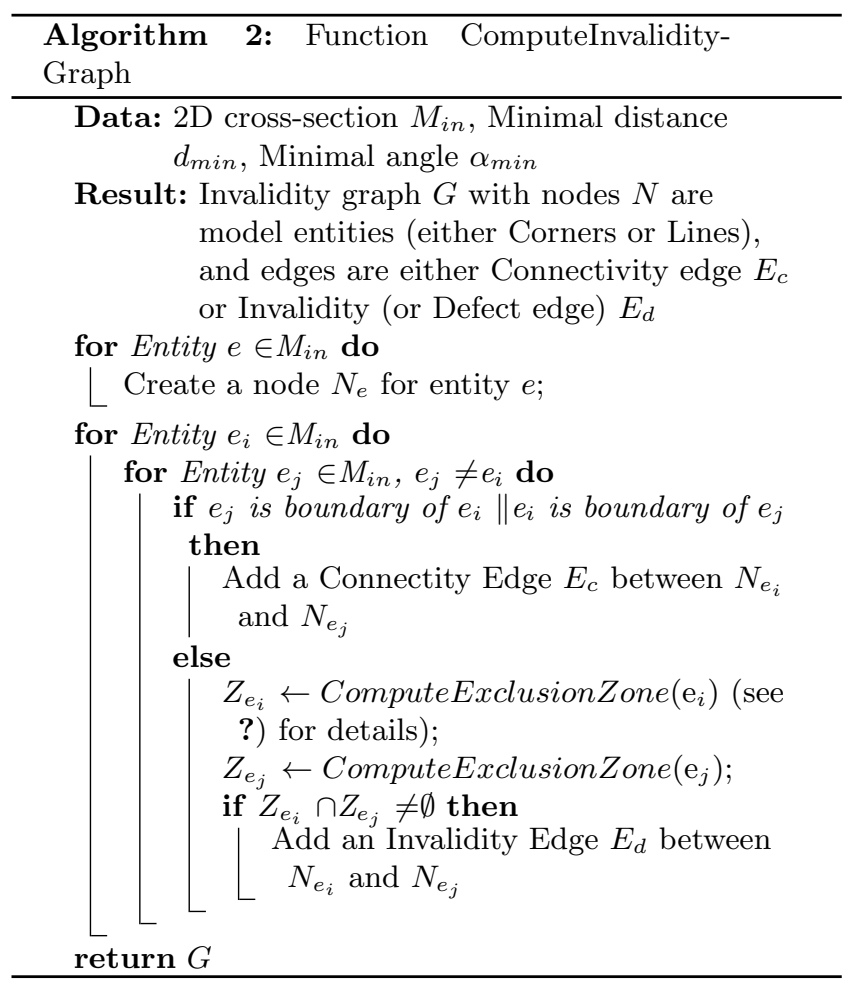



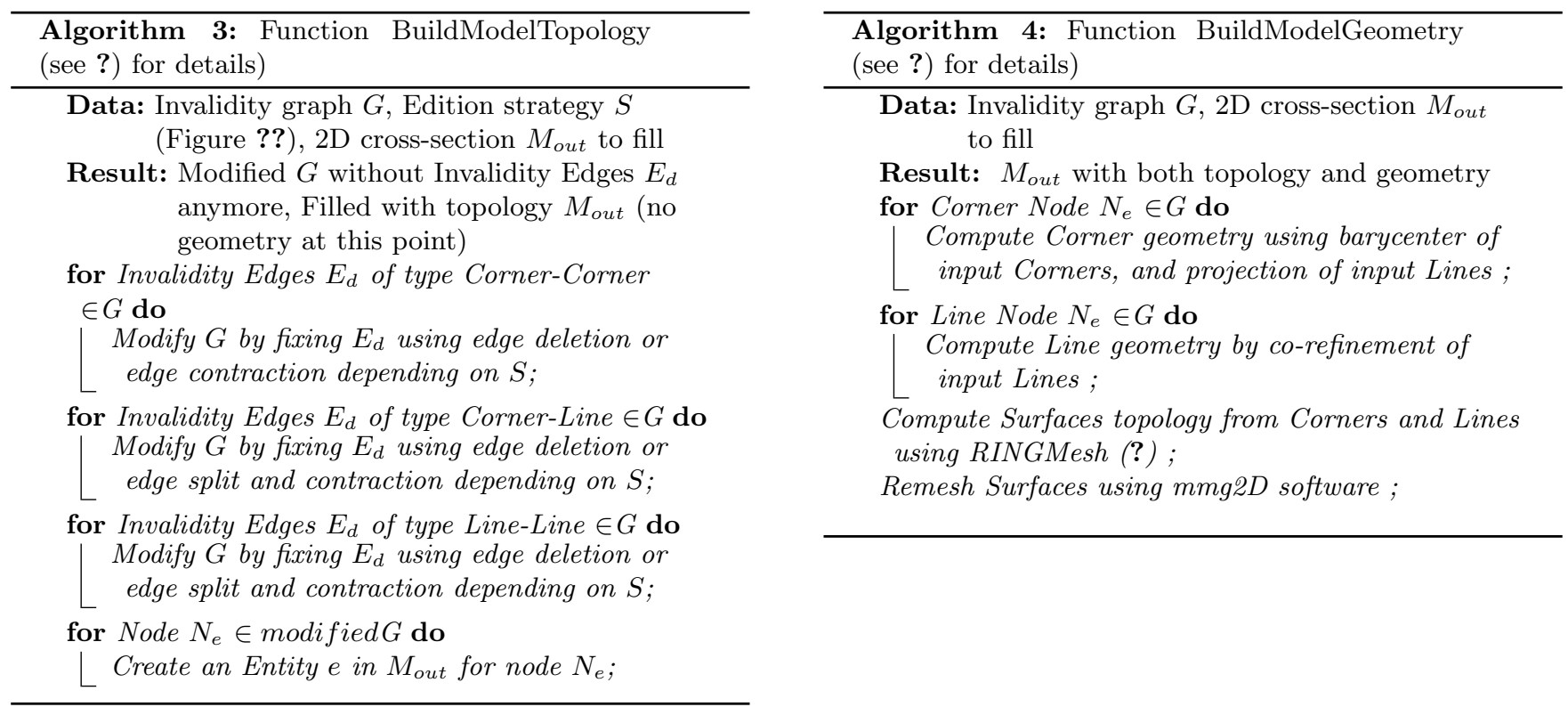

\section{REFERENCES}

Anquez, P., 2019. Correction et simplification de modèles géologiques par frontières: impact sur le maillage et la simulation numérique en sismologie et hydrodynamique, Ph.D. thesis, Université de Lorraine.

Anquez, P., Pellerin, J., Irakarama, M., Cupillard, P., Lévy, B., \& Caumon, G., 2019. Automatic correction and simplification of geological maps and cross-sections for numerical simulations, Comptes Rendus Geoscience, 351(1), 48-58.

Bard, P.-Y., 1997. Local effects on strong ground motion: Basic physical phenomena and estimation methods for microzoning studies, Advanced study course on Seismik Risk (SERINA), Thessaloniki, Greece, pp. 229-299.

Bard, P.-Y. \& Bouchon, M., 1980a. The seismic response of sediment-filled valleys. Part 1 - The case of incident SH waves, Bulletin of the Seismological Society of America, 70(4), 12631286.

Bard, P.-Y. \& Bouchon, M., 1980b. The seismic response of sediment-filled valleys. Part 2 - The case of incident P and SV waves, Bulletin of the Seismological Society of America, 70(5), 1921-1941.

Baudouin, T., Remacle, J.-F., Marchandise, E., Henrotte, F., \& Geuzaine, C., 2014. A frontal approach to hex-dominant mesh generation, 1(1), 8 .

Bertrand, E., Duval, A., Castan, M., \& Vidal, S., 2007. 3D geotechnical soil model of Nice, France, inferred from seismic noise measurements for seismic hazard assessment, in $A G U$ Fall Meeting, San Francisco.

Bielak, J., MacCamy, R. C., McGhee, D. S., \& Barry, A., 1991. Unified symmetric BEM-FEM for site effects on ground motion - SH waves, Journal of Engineering Mechanics, 117(10), 22652285.

Bielak, J., Xu, J., \& Ghattas, O., 1999. Earthquake ground motion and structural response in alluvial valleys, Journal of Geotechnical and Geoenvironmental Engineering, 125(5), 413423.

Bielak, J., Ghattas, O., \& Kim, E., 2005. Parallel octree-based finite element method for large-scale earthquake ground motion simulation, Computer Modeling in Engineering and Sciences, 10(2), 99-112.

Borcherdt, R. \& Gibbs, J., 1970. Effects of local geological conditions in the San Francisco Bay region on ground motions and the intensities of the 1906 earthquake, Bulletin of the Seismological Society of America, 66, 467-500.

Botella, A., Lévy, B., \& Caumon, G., 2016. Indirect unstruc- 
tured hex-dominant mesh generation using tetrahedra recombination, Computational Geosciences, 20(3), 437-451.

Bouchon, M., Campillo, M., \& Gaffet, S., 1989. A boundary integral equation-discrete wavenumber representation method to study wave propagation in multilayered media having irregular interfaces, Geophysics, 54(9), 1134-1140.

Bourgine, B., 2018. GDM, Outils Géostatistiques - Manuel de référence, BRGM, Orléans, France.

Brocher, T., 2008. Key elements of seismic velocity models for long period ground motion simulations, Journal of Seismology, $12(2), 217-221$.

Campillo, M. \& Bouchon, M., 1985. Synthetic SH seismograms in a laterally varying medium by the discrete wavenumber method, Geophysical Journal International, 83(1), 307-317.

Capdeville, Y. \& Marigo, J.-J., 2008. Shallow layer correction for spectral element like methods, Geophysical Journal International, 172(3), 1135-1150.

Capdeville, Y., Guillot, L., \& Marigo, J., 2010. 2-D non-periodic homogenization to upscale elastic media for P-SV waves, Geophysical Journal International, 182(2), 903-922.

Capdeville, Y., Cupillard, P., \& Singh, S., 2020. An introduction to the homogenization theory for seismology, Advances in Geophysics, accepted.

Casadei, F., Gabellini, E., Fotia, F., Maggio, F., \& Quarteroni, A., 2002. A mortar spectral/finite element method for complex 2D and 3D elastodynamic problems, Computer Methods in Applied Mechanics and Engineering, 191, 5119-5148.

Catuneanu, O., 2019. Scale in sequence stratigraphy, Marine and Petroleum Geology, 106, 128-159.

Caumon, G., 2018. Geological objects and physical parameter fields in the subsurface: A review, in Handbook of Mathematical Geosciences, pp. 567-588, eds Daya Sagar, B., Cheng, Q., \& Agterberg, F., Springer International Publishing.

Chabassier, J. \& Imperiale, S., 2016. Fourth order energy preserving locally implicit time-discretization for linear wave equations, International Journal for Numerical Methods for Engineering, 106(8), 593-622.

Chaillat, S., Bonnet, M., \& Semblat, J.-F., 2009. A new fast multi-domain BEM to model seismic wave propagation and amplification in 3-D geological structures, Geophysical Journal International, $\mathbf{1 7 7}(2), 509-531$.

Chaljub, E., Capdeville, Y., \& Vilotte, J.-P., 2003. Solving elastodynamics in a fluid-solid heterogeneous sphere: a parallel spectral element approximation on non-conforming grids, Journal of Computational Physics, 187(2), 457-491.

Chaljub, E., Moczo, P., Tsuno, S., Bard, P.-Y., Kristek, J., Käser, M., Stupazzini, M., \& Kristekova, M., 2010. Quantitative comparison of four numerical predictions of 3D ground motion in the Grenoble Valley, France, Bulletin of the Seismological Society of America, 100(4), 1427-1455.

Chávez-Garcia, F. \& Faccioli, E., 2000. Complex site effects and building codes: making the leap, Journal of Seismology, 4, 23-40.

Chávez-Garcia, F., Raptkis, D., Makra, K., \& Pitilakis, K., 2000. Site effects at Euroseistest - II. Results from 2D numerical modeling and comparison with observations, Soil Dynamics and Earthquake Engineering, 19, 23-39.

Chin-Joe-Kong, M., Mulder, W., \& van Veldhuizen, M., 1999. Higher-order triangular and tetrahedal finite elements with mass lumping for solving the wave equation, Journal of Engineering Mathematics, 35, 405-426.

Cruz-Atienza, V. M., Tago, J., Sanabria-Gómez, J. D., Chaljub, E., Etienne, V., Virieux, J., \& Quintanar, L., 2016. Long Duration of Ground Motion in the Paradigmatic Valley of Mexico, Scientific Reports, 6(1), 38807.

Cupillard, P. \& Capdeville, Y., 2018. Non-periodic homogenization of 3-D elastic media for the seismic wave equation, Geophysical Journal International, 213(2), 983-1001.
Cupillard, P., Delavaud, E., Burgos, G., Festa, G., Vilotte, J.-P., Capdeville, Y., \& Montagner, J.-P., 2012. RegSEM: a versatile code based on the spectral element method to compute seismic wave propagation at the regional scale, Geophysical Journal International, 188(3), 1203-1220.

Cupillard, P., Mulder, W., Anquez, P., Mazuyer, M., \& Barthélémy, J.-F., 2020. The apparent anisotropy of the SEGEAGE overthrust model, in 82nd EAGE Conference and Exhibition, European Association of Geoscientists \& Engineers, accepted.

Dapogny, C., Dobrzynski, C., \& Frey, P., $2014 . \quad$ Threedimensional adaptive domain remeshing, implicit domain meshing, and applications to free and moving boundary problems, Journal of Computational Physics, 262, 358-378.

Daya Sagar, B. S., 2018. Mathematical morphology in geosciences and GISci: An illustrative review, in Handbook of Mathematical Geosciences: Fifty Years of IAMG, pp. 703-740, eds Daya Sagar, B., Cheng, Q., \& Agterberg, F., Springer International Publishing, Cham.

De Martin, F., 2011. Verification of a spectral-element code for the Southern California Earthquake Center LOH.3 viscoelastic case, Bulletin of the Seismological Society of America, 101(6), 2855-2865.

Delavaud, E., Cupillard, P., Festa, G., \& Vilotte, J. P., 2006. 3D Spectral Element Method simulations of the seismic response in the Caracas basin, in Proc. of the Third International Symposium on the Effects of Surface Geology on Seismic Motion, vol. 1, pp. 515-522, Grenoble, France.

Delcourte, S. \& Glinsky, N., 2015. Analysis of a high-order space and time discontinuous Galerkin method for elastodynamic equations. Application to 3D wave propagation, ESAIM Mathematical Modelling and Numerical Analysis, 49(4), 10851126.

Diaz, J. \& Grote, M., 2015. Multi level explicit local timestepping methods for second-order wave equations, Computer Methods in Applied Mechanics and Engineering, 291, 240-265. Diaz, J. \& Joly, P., 2005. Robust high order non-conforming finite element formulation for time domain fluid-structure interaction, Journal of computational acoustics, 13(03), 403-431.

Dobrzynski, C. \& Frey, P., 2008. Anisotropic Delaunay Mesh Adaptation for Unsteady Simulations, in Proceedings of the 17th International Meshing Roundtable, pp. 177-194, ed. Garimella, R. V., Springer Berlin Heidelberg, Berlin, Heidelberg.

Dumbser, M. \& Käser, M., 2006. An arbitrary high-order Discontinuous Galerkin method for elastic waves on unstructured meshes - II. The three-dimensional isotropic case, Geophysical Journal International, 167(1), 319-336.

Dumbser, M., Käser, M., \& Toro, E. F., 2007. An arbitrary high-order Discontinuous Galerkin method for elastic waves on unstructured meshes - V. Local time stepping and p-adaptivity, Geophysical Journal International, 171(2), 695-717.

Emmerich, H. \& Korn, M., 1987. Incorporation of attenuation into time-domain computations of seismic wave fields, Geophysics, 52(9), 1252-1264.

Etienne, V., Chaljub, E., Virieux, J., \& Glinsky, N., 2010. An hp-adaptive discontinuous Galerkin finite-element method for 3-D elastic wave modelling, Geophysical Journal International, 183(2), 941-962.

Festa, G., Delavaud, E., \& Vilotte, J. P., 2005. Interaction between surface waves and absorbing boundaries for wave propagation in geological basins: 2D numerical simulations, Geophys. Res. Letters, 32, L20306, doi:10.1029/2005GL024091.

Field, E. \& Jacob, K., 1995. A comparison and test of various site-response estimation techniques, including three that are not reference-site dependent, Bulletin of the Seismological Society of America, 85, 1127-1143.

Frankel, A., Hough, S., Friberg, P., \& Busby, R., 1991. Ob- 
servations of Loma Prieta afteshocks from a dense array in Sunnyvale, California, Bulletin of the Seismological Society of America, 81(5), 1900-1922.

Glinsky, N., Bertrand, E., \& Régnier, J., 2019. Numerical simulation of topographical and geological site effects. Applications to canonical topographies and Rognes hill, South East France, Soil Dynamics and Earthquake Engineering, 116, 620-636.

Gomes, A. T., Paredes, D., Pereira, W., Souto, R., \& Valentin, F., 2017. A Multiscale Hybrid-Mixed Method for the Elastodynamic Model with Rough Coefficients, in Proceedings of the XXXVIII Iberian Latin American Congress of Computational Methods in Engineering, \{ABMEC\} Brazilian Association of Computational Methods in Engineering.

Gravouil, A. \& Combescure, A., 2001. Multi-time-step explicitimplicit method for non-linear structural dynamics, International Journal for Numerical Methods in Engineering, 50(1), 199-225.

Guidotti, R., Stupazzini, M., Smerzini, C., Paolucci, R., \& Ramieri, P., 2011. Numerical study on the role of basin geometry and kinematic seismic source in 3D ground motion simulation of the 22 February $2011 \mathrm{Mw}$ 6.2 Christchurch earthquake, Seismological Research Letters, 82(6), 767-782.

Guillot, L., Capdeville, Y., \& Marigo, J., 2010. 2-D non-periodic homogenization of the elastic wave equation: SH case, Geophysical Journal International, 182(3), 1438-1454.

Gutenberg, B., 1957. Effects of ground on earthquake motion, Bulletin of the Seismological Society of America, 47(3), 221250 .

Hermann, V., Käser, M., \& Castro, C. E., 2011. Non-conforming hybrid meshes for efficient 2-D wave propagation using the Discontinuous Galerkin Method, Geophysical Journal International, 184(2), 746-758.

Ichimura, T., Hori, M., \& Bielak, J., 2009. A hybrid multiresolution meshing technique for finite element three-dimensional earthquake ground motion modelling in basins including topography, Geophysical Journal International, 177(3), 1221-1232.

Kamal \& Narayan, J., 2016. Study of effects of sedimentdamping, impedance contrast, and size of semi-spherical basin on the focusing and trapping on the basin-generated surface waves, Journal of Earthquake Engineering, 20(3), 406-427.

Käser, M. \& Dumbser, M., 2006. An arbitrary high-order discontinuous Galerkin method for elastic waves on unstructured meshes - I. The two-dimensional isotropic case with external source terms, Geophysical Journal International, 166(2), 855877 .

Kawase, H., 1996. The Cause of the Damage Belt in Kobe: "The Basin-Edge Effect", Constructive Interference of the Direct SWave with the Basin-Induced Diffracted/Rayleigh Waves, Seismological Research Letters, 67(5), 25-34.

Kawase, H. \& Aki, K., 1989. A study on the response of a soft basin for incident S, P, and Rayleigh waves with special reference to the long duration observed in Mexico City, Bulletin of the Seismological Society of America, 79(5), 1361-1382.

Kawase, H. \& Aki, K., 1990. Topography effect at the critical SV-wave incidence: possible explanation of damage pattern by the Whittier Narrows, California, earthquake of 1 October 1987, Bulletin of the Seismological Society of America, 80(1), $1-22$.

Klin, P., Priolo, E., \& Seriani, G., 2010. Numerical simulation of seismic wave propagation in realistic $3-\mathrm{D}$ geo-models with a Fourier pseudo-spectral method, Geophysical Journal International, 183(2), 905-922.

Komatitsch, D. \& Vilotte, J.-P., 1998. The spectral element method: An efficient tool to simulate the seismic response of 2D and 3D geological structures, Bulletin of the Seismological Society of America, 88(2), 368-392.

Komatitsch, D., Liu, Q., Tromp, J., Suss, P., Stidham, C., \& Shaw, J. H., 2004. Simulations of ground motion in the Los
Angeles basin based upon the spectral-element method, Bulletin of the Seismological Society of America, 94(1), 187-206. Le Bun, B., Hatzfeld, D., Bard, P.-Y., \& Bouchon, M., 1999. Experimental study of the ground motion on a large scale topographic hill at Kitherion (Greece), Journal of Seismology, 3(1), 1-15.

Lovati, S., Bakavoli, M., Massa, M., Ferretti, G., Pacor, F., Paolucci, R., Haghshenas, E., \& Kamalian, M., 2011. Estimation of topographical effects at Narni ridge (Central Italy): comparisons between experimental results and numerical modelling, Bulletin of Earthquake Engineering, 9(6), 1987-2005.

Makra, K. \& Chávez-Garcia, F., 2016. Site effects in 3D basins using 1D and 2D models: an evaluation of the differences based on simulations of the seismic response of Euroseistest, Bull. Earthquake Eng., 14, 1177-1194.

Mandad, M., Cohen-Steiner, D., \& Alliez, P., 2015. Isotopic approximation within a tolerance volume, ACM Transactions on Graphics, 34(4), 64:1-64:12.

Martino, S., Lenti, L., Gélis, C., Giacomi, A., Santisi d'Avila, M., Bonilla, L., Bozzano, F., \& Semblat, J.-F., 2015. Influence of lateral heterogeneities on strong-motion shear strains: Simulations in the historical center of Rome (Italy), Bulletin of the Seismological Society of America, 105(5), 2604-2624.

Massa, M., Barani, S., \& Lovati, S., 2014. Overview of topographic effects based on experimental observations: meaning, causes and possible interpretation, Geophysical Journal International, $197(3)$, 1551-1565.

Maufroy, E., Chaljub, E., Hollender, F., Kristek, J., Moczo, P., Klin, P., Priolo, E., Iwaki, A., Iwata, T., Etienne, V., De Martin, F., Theodoulidis, N., Manakou, M., Guyonnet-Benaize, C., Pitilakis, K., \& Bard, P.-Y., 2015. Earthquake ground motion in the Mygdonian basin, Greece: The E2VP Verification and Validation of 3D numerical simulation up to $4 \mathrm{~Hz}$, Bulletin of the Seismological Society of America, 105(3), 1398-1418.

Mazzieri, I., Stupazzini, M., Guidotti, R., \& Smerzini, C., 2013. SPEED: SPectral Elements in Elastodynamics with Discontinuous Galerkin: A non-conforming approach for 3D multi-scale problems, International Journal for Numerical Methods in Engineering, 95(12), 991-1010.

Mercerat, E. D. \& Glinsky, N., 2015. A nodal high-order discontinuous Galerkin method for elastic wave propagation in arbitrary heterogeneous media, Geophysical Journal International, 201(2), 1101-1118.

Meza-Fajardo, K., Varone, C., Lenti, L., \& Martino, S., 2019. Surface wave quantification in a highly heterogeneous alluvial basin: Case study of the Fosso di Vallerano valley, Rome, Italy, Soil Dynamics and Earthquake Engineering, 120, 292-300.

Moczo, P., Bystrickỳ, E., Kristek, J., Carcione, J. M., \& Bouchon, M., 1997. Hybrid modeling of P-SV seismic motion at inhomogeneous viscoelastic topographic structures, Bulletin of the Seismological Society of America, 87(5), 1305-1323.

Moczo, P., Kristek, J., \& Gális, M., 2014. The finite-difference modelling of earthquake motions: waves and ruptures, Cambridge University Press, Cambridge.

Moczo, P., Kristek, J., Bard, P.-Y., Stripajová, S., Hollender, F., Chovanová, Z., Kristeková, M., \& Sicilia, D., 2018. Key structural parameters affecting earthquake ground motion in 2D and 3D sedimentary structures, Bull. Earthquake Eng., 16, 2421-2450.

Narayan, J., Singh, P., \& Kamal, 2016. Effects of basin parameters on the spatial variation of characteristics of basin generated Rayleigh waves, International Journal of Geo-Engineering, 7, 17.

Olsen, K. B., 2000. Site amplification in the Los Angeles basin from three-dimensional modeling of ground motion, Bulletin of the Seismological Society of America, 90(6B), S77-S94.

Pellerin, J., Botella, A., Bonneau, F., Mazuyer, A., Chauvin, B., Lévy, B., \& Caumon, G., 2017. RINGMesh: A programming 
library for developing mesh-based geomodeling applications, Computers \& Geosciences, 104, 93-100.

Peyrusse, F., Glinsky, N., Gélis, C., \& Lanteri, S., 2014. A nodal discontinuous Galerkin method for site effects assessment in viscoelastic media Verification and validation in the Nice basin, Geophysical Journal International, 199(1), 315-334.

Restrepo, D., Bielak, J., Serrano, R., Gómez, J., \& Jaramillo, J., 2016. Effects of realistic topography on the ground motion of the Colombian Andes - A case study at the Aburrá Valley, Antioquia, Geophysical Journal International, 204(3), 18011816.

Rietmann, M., Grote, M., Peter, D., \& Schenk, O., 2017. Newmark local time stepping on high-performance computing architectures, Journal of Computational Physics, 334, 308-326. Riga, E., Makra, K., \& Pitilakis, K., 2016. Aggravation factors for seismic response of sedimentary basins: A code-oriented parametric study, Soil Dynamics and Earthquake Engineering, 91, 116-132.

Ringrose, P. S., Martinius, A. W., \& Alvestad, J., 2008. Multiscale geological reservoir modelling in practice, Geological Society, London, Special Publications, 309(1), 123-134.

Rohmer, O., Bertrand, E., Mercerat, E., Régnier, J., Pernoud, M., Langlaude, P., \& Alvarez, M., 2020. Combining borehole log-stratigraphies and ambient vibration data to build a 3D model of the Lower Var Valley, Nice (France), Engineering Geology, 270, 105588.

Salichon, J., Kohrs-Sansorny, C., Bertrand, E., \& Courboulex, F., 2010. A Mw 6.3 earthquake scenario in the city of Nice (southeast France): ground motion simulations, Journal of Seismology, 14(3), 523-541.

Sánchez-Sesma, F. J. \& Campillo, M., 1993. Topographic effects for incident P, SV and Rayleigh waves, Tectonophysics, 218(1), 113-125.

Sánchez-Sesma, F. J. \& Luzón, F., 1995. Seismic response of three-dimensional alluvial valleys for incident P, S, and Rayleigh waves, Bulletin of the Seismological Society of America, 85(1), 269-284.

Sato, T., Graves, R. W., \& Somerville, P. G., 1999. Threedimensional finite-difference simulations of long-period strong motions in the Tokyo metropolitan area during the 1990 Odawara earthquake (MJ 5.1) and the great 1923 Kanto earthquake (MS 8.2) in Japan, Bulletin of the Seismological society of America, 89(3), 579-607.

Semblat, J.-F., Duval, A.-M., \& Dangla, P., 2000. Numerical analysis of seismic wave amplification in Nice (France) and comparisons with experiments, Soil Dynamics and Earthquake Engineering, 19(5), 347-362.

Serra, J., 1969. Introduction à la morphologie mathématique, Centre de morphologie mathématique de Fontainebleau.

Serra, J., 1983. Image analysis and mathematical morphology, Academic Press, Inc.

Serra, J., 1986. Introduction to mathematical morphology, Computer vision, graphics, and image processing, 35(3), 283-305. Shani-Kadmiel, S., Tsesarsky, M., Louie, J., \& Gvirtzman, Z., 2012. Simulation of seismic-wave propagation through geometrically complex basins: The Dead Sea Basin, Bulletin of the Seismological Society of America, 102(4), 1729-1739.

Singh, S. K., Mena, E., \& Castro, R., 1988. Some aspects of source characteristics of the 19 September 1985 Michoacan earthquake and ground motion amplification in and near Mexico City from strong motion data, Bulletin of the Seismological Society of America, 78(2), 451-477.

Smerzini, C. \& Paolucci, R., 2011. Comparison of 3D, 2D and 1D numerical approaches to predict long period earthquake ground motion in the Gubbio plain, Central Italy, Bull. Earthquake Eng., 9, 2007-2029.

Sochala, P., Martin, F. D., \& Matre, O. L., 2020. Model reduction for large-scale earthquake simulation in an uncertain 3d medium, International Journal for Uncertainty Quantification, 10(2), 101-127.

Straub, K. M. \& Foreman, B. Z., 2018. Geomorphic stasis and spatiotemporal scales of stratigraphic completeness, Geology, 46(4), 311-314.

Stupazzini, M., Paolucci, R., \& Igel, H., 2009. Near-fault earthquake ground motion simulation in the Grenoble Valley by a high performance spectral element code, Bulletin of the Seismological Society of America, 99, 286-301.

Terrana, S., Vilotte, J. P., \& Guillot, L., 2017. A spectral hybridizable discontinuous Galerkin method for elastic-acoustic wave propagation, Geophysical Journal International, 213(1), 574-602.

Thompson, M., Wirth, E., Frankel, A., Hartog, J., \& Vidale, J., 2020. Basin amplification effects in the Puget Lowland, Washington, from strong-motion recordings and 3D simulations, Bulletin of the Seismological Society of America, 110(2), $534-555$.

Vidale, J. E. \& Helmberger, D. V., 1988. Elastic finite-difference modeling of the 1971 San Fernando, California earthquake, Bulletin of the Seismological Society of America, 78(1), 122141 .

Wellmann, F. \& Caumon, G., 2018. 3-D Structural geological models: Concepts, methods, and uncertainties, Advances in Geophysics, 59, 1-121.

Wilcox, L. C., Stadler, G., Burstedde, C., \& Ghattas, O., 2010. A high-order discontinuous Galerkin method for wave propagation through coupled elastic-acoustic media, Journal of Computational Physics, 229(24), 9373-9396.

Yamakawa, S. \& Shimada, K., 2003. Fully-automated hexdominant mesh generation with directionality control via packing rectangular solid cells, International Journal for Numerical Methods in Engineering, 57(15), 2099-2129.

Yoshimura, C., Bielak, J., Hisada, Y., \& Fernández, A., 2003. Domain reduction method for three-dimensional earthquake modeling in localized regions, part II: Verification and applications, Bulletin of the Seismological Society of America, 93(2), 825-841.

Zhao, M., Capdeville, Y., \& Zhang, H., 2016. Direct numerical modeling of time-reversal acoustic subwavelength focusing, Wave Motion, 67, 102-115. 